\title{
Accelerating AUTODoCK4 with GPUs and Gradient-Based Local Search
}

\author{
Diogo Santos-Martins $^{1, \mathrm{a}}$, Leonardo Solis-Vasquez ${ }^{1, \mathrm{~b}}$, Andreas $\mathrm{Koch}^{\mathrm{b}, \mathbb{\varpi}}$, and Stefano Forli ${ }^{\mathrm{a}, \mathbb{}}$ \\ ${ }^{1}$ These authors contributed equally to this work. \\ ${ }^{a}$ Department of Integrative Structural and Computational Biology, The Scripps Research Institute, La Jolla, California, USA \\ ${ }^{\mathrm{b}}$ Embedded Systems and Applications Group, Technische Universität Darmstadt, Germany
}

\begin{abstract}
AuTODock4 is a widely used program for docking small molecules to macromolecular targets. It describes ligandreceptor interactions using a physics-inspired scoring function that has been proven useful in a variety of drug discovery projects. However, compared to more modern and recent software, AUTODock4 has longer execution times, limiting its applicability to large scale dockings. To address this problem, we describe an OpenCL implementation of AUTODock4, called AUTODocK-GPU, that leverages the highly parallel architecture of GPU hardware to improve the docking throughput up to 170-fold. Moreover, we introduce the gradient-based local search method ADADELTA, which is more efficient than the original Solis-Wets method, especially for conformationally complex ligands. We estimate a $1300 x$ reduction in the number of scoring function calls for ligands with 20 rotatable bonds, and even higher reductions likely for more complex ligands. The improvements reported here, both in terms of docking throughput and search efficiency, expand the domain of applicability of the AUTODOCK4 scoring function considerably.
\end{abstract}

Molecular Docking | AutoDock | High Performance Computing | GPU

Correspondence: koch@esa.tu-darmstadt.de forli@scripps.edu

\section{INTRODUCTION}

The identification of new molecules with a given biological activity is a non-trivial task, and a key step in the drug discovery process. Structure-based virtual screening in which small organic molecules are docked to biologically and therapeutically relevant targets is a very popular computational technique for the identification of new chemical scaffolds with biological activity.

Over the years, this technique has provided numerous positive results competitive with experimental screenings (1-5). The two main factors contributing to its success is the use of energy estimate methods (or scoring functions) that are sufficiently accurate to distinguish (to a certain extent) between binders and non-binders, while being sufficiently fast to be applicable on a very large scale. Typical virtual screenings now can easily involve virtual libraries of compounds with hundred thousand to several million molecules against relatively rigid target structures. Depending on the search algorithms, this translates in $10^{10}$ to $10^{16}$ calls to the scoring function. The increasing availability of targets from structural genomics initiatives (6), as well as the use of target conformational ensembles (7) to address receptor conformational variability, can easily add three or more orders of mag- nitude.

Molecular docking is particularly suitable for parallel computing, given the embarrassingly parallel nature of the tasks involved. Scoring function and search calls can be easily distributed across multiple computing units, either being CPU cores on the same machine (8), or many CPU nodes across multiple machines in high-performance computing (HPC) environments (9), or distributed computing resources $(10,11)$. From that perspective, GPU computing represents a hybrid scenario, in which a large number of computing units (i.e., GPU cores) can be accessed on a single machine. The availability of GPU cards with thousands cores represents a cheap but powerful computational resource that has already been exploited to accelerate a number of molecular modeling computations (12-14).

AUTODOCK is a widely used molecular docking program. From a computational standpoint, it is characterized by its single-threaded behavior, which means it only utilizes a single CPU core during execution. By exploiting the underlying algorithms' embarrassingly parallel nature, its efficient adaption to accelerators can result in significant performance improvements. Expressing such parallelism in a suitable manner can be achieved through parallel programming frameworks (15-17). One of these, the Open Computing Language - OpenCL - provides an open standard that is portable across hybrid platforms such as CPUs and GPUs. From an algorithmic standpoint, and based on the potential gains from an OpenCL implementation, more complex search methods can be explored without incurring the big performance penalties that would affect the single-threaded AUTODOCK version.

In this work, we describe our OpenCL implementation of AUTODOCK for GPUs. This program is based on an improved version of the AUTODOCK search algorithm, which introduces scoring-function gradients for translation, rotation, and torsion variables. For global search, it still employs a Lamarckian Genetic Algorithm (LGA) similar to the one used in AUTODocK (since version 4.0) (18). For the local search, we newly incorporate the ADADELTA (19) gradientbased method, while also including the original random numerical optimizer Solis-Wets (20). Through a number of experiments, we evaluated the computational and algorithmic enhancements provided by our OpenCL implementation over the original AUTODOCK. 


\section{METHODOLOGY}

Brief introduction to AUTODock docking. In

AUTODосK, molecular interactions are modeled by a scoring function $f$ that quantifies the free energy of a given binding pose (21):

$$
\begin{array}{r}
f=W_{\mathrm{vdw}} \sum_{i, j}\left(\frac{A_{i j}}{r_{i j}^{12}}-\frac{B_{i j}}{r_{i j}^{6}}\right)+W_{\mathrm{hb}} \sum_{i, j} E(t)\left(\frac{C_{i j}}{r_{i j}^{12}}-\frac{D_{i j}}{r_{i j}^{10}}\right)+ \\
W_{\mathrm{el}} \sum_{i, j}\left(\frac{q_{i} q_{j}}{\epsilon\left(r_{i j}\right) r_{i j}}\right)+W_{\mathrm{ds}} \sum_{i, j}\left(S_{i} V_{j}+S_{j} V_{i}\right) e^{\frac{-r_{i j}^{2}}{2 \sigma^{2}}}+ \\
W_{\text {rot }} N_{\text {rot }}
\end{array}
$$

The AuToDock scoring function (Equation 1) is a semiempirical free-energy force field $(\mathrm{kcal} / \mathrm{mol})$ composed of four pairwise energetic terms (dispersion/repulsion, hydrogen bonding, electrostatics, and desolvation), and an additional term that predicts the (unfavorable) loss of ligand entropy upon binding. The dimensionless weighting constants $W_{\mathrm{vdw}}, W_{\mathrm{hb}}, W_{\mathrm{el}}, W_{\mathrm{ds}}$, and $W_{\text {rot }}$ were empirically determined using linear regression on a set of ligand-receptor complexes with known binding constants. The following constants depend on the atom types: $A_{i j}\left(\mathrm{kcal} / \mathrm{mol} \AA^{12}\right)$ and $B_{i j}\left(\mathrm{kcal} / \mathrm{mol} \AA^{6}\right)$ correspond to the Lennard-Jones (12-6) potential between atoms $i$ and $j ; C_{i j}\left(\mathrm{kcal} / \mathrm{mol} \AA^{12}\right)$ and $D_{i j}$ ( $\mathrm{kcal} / \mathrm{mol} \AA^{10}$ ) correspond to the hydrogen bonding (12-10) potential between hydrogen-bond acceptor and donor atoms $i$ and $j ; S$ and $V$ are respectively the solvation parameter and the atom volume that shelters it from the solvent, while $\sigma=$ $3.5 \AA$ is an independent constant. The $E(t)$ function represents the dimensionless directional weight of the angle $t$ that provides directionality from ideal hydrogen bonding geometry. Additionally, $q_{i}$ and $q_{j}$ are atomic charges, while $\epsilon\left(r_{i j}\right)$ is a dielectric function of $r_{i j}$, the interatomic distance between atoms $i$ and $j$. The last term, proportional to the number of torsions $N_{\text {rot }}$, measures the unfavorable loss of ligand entropy due to the restriction of conformational degrees of freedom in the bound state.

The overall molecular interaction can be expressed as the sum of two independent interactions based on ligand and receptor group of atoms. Intermolecular interactions (i.e., ligand-receptor) are precalculated and cached using the gridbased method of the AUTOGRID program, which calculates interaction energy maps for all ligand atoms, with a default resolution of $0.375 \AA$. These maps are used to lookup interaction energies using trilinear interpolation, speeding up intermolecular interaction estimates compared to pairwise methods. Similarly, in the AutoDock4 implementation, intramolecular interactions (i.e., ligand-ligand) are precalculated for all atom pairs and stored in one-dimensional look-up tables.

A Lamarckian Genetic Algorithm (LGA) is used to generate ligand poses exploring the energy landscape described by the scoring function (22). The LGA combines a global search based on a genetic algorithm (GA) - with local search (LS) iterations to refine poses identified by the GA. Poses that are improved by the LS method are re-introduced into the GA population (hence the Lamarckian denomination). All calculated poses form a population, where each member, i.e., an individual, is represented by a vector of genes (i.e., genotype). Each gene represents a specific ligand motion, controlling the conformation and orientation of the ligand. New individuals are generated by the GA from ancestors chosen with a proportional selection scheme. A population subset (default: 6\%) is subjected to the LS optimization, which in AuTODock4 is based on the Solis-Wets method (20). The LGA execution stops when a pre-defined number of score evaluations or GA generations is reached, whichever comes first.

Furthermore, a docking job consists of several independent LGA runs (default: 50). Each LGA run optimizes the sum of intermolecular (ligand-receptor) and intramolecular (ligandligand) interactions described by the scoring function $f$, and returns the best pose (lowest score) it could find. After all LGA runs are complete, the returned poses are clustered using the root mean square deviation (RMSD) as distance metric. The size of the cluster containing the pose with the best score can be used as a proxy to identify convergence of the LGA runs towards a particular solution (pose) within the search space. As a general rule, the search effort is considered sufficient if the first cluster contains about $20 \%$ (or more) of poses returned by LGA runs.

OpenCL implementation of AUTODocK. As both GA and LS optimizations require many scoring function calls for a single docking job (default: in the order of million evaluations), these optimizations become the bottleneck accounting together for more than $90 \%$ of the entire application runtime (23). Therefore, offloading these optimization methods onto an accelerator, like a GPU, can profit significantly from the speedups enabled by more specialized hardware.

The improvements of the AUTODOCK search algorithm introduced in this paper were built on top of our previous work (23), released as the open-source software project OpenCL Accelerated Molecular Docking, hereafter simply referred to as AUTODocK-GPU (24). In this section, we describe various software-related aspects of our implementation, starting with OpenCL basics, going through our parallelization approach, as well as providing insights of the main design considerations made in this work.

OpenCL background. Programming frameworks are essential to leverage the performance capabilities of highlyparallel computing platforms (15-17). In that regard, OpenCL provides an open, and royalty-free standard for writing parallel programs that execute across heterogeneous platforms consisting of CPUs, GPUs, and other hardware accelerators, providing software developers with a portable abstraction for accelerating computationallyintensive tasks (25). 
A brief introduction to the OpenCL programing model is presented as follows. An OpenCL platform is a vendor-specific implementation of the standard, and defines the logical representation of the hardware capable of executing an OpenCL application. Basically, an OpenCL platform is composed of two main elements, i.e., a host processor, and a number of computing devices, both being elements connected through a bus. The host is responsible for the general platform management, while the device is in charge of executing clearly-defined functions in parallel, which are called kernels. These kernels are described using OpenCL C, which is a C-like language with extensions for parallel programming such as multithreading and synchronization mechanisms. A kernel can be described as a function of indices (Figure 1). Each index-based kernel element is called a work-item (wi), which can be thought of as a processing thread. Moreover, the total number of indices is partitioned into work-groups (WG). Communication between individual work-items within a work-group is possible and can be achieved through global or locally-shared memory, while their synchronization can be enforced through barrier operations.
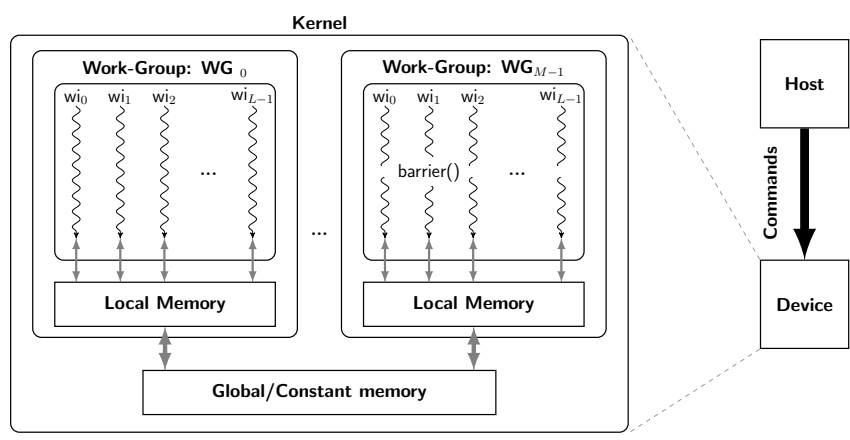

Fig. 1. An OpenCL platform is composed of a host and one or more devices. The host controls the device(s) via commands. A device is in charge of executing kernels, each of which can be subdivided into $M$ work-groups (WGs). Each work-group can be further subdivided into $L$ work-items (wis). Within a work-group, communication and synchronization between work-items is possible using local memory and barrier operations, respectively.

Parallelization opportunities and OpenCL strategy. As reported in a previous study (26), data parallelism of AuTODocK is exploited at three different processing levels: high, medium, and low. First, based on the fact that a docking job consists of several independent LGA runs (default: 50), the high-level parallelization consists of trivially performing these runs in parallel. Second, individuals from a single genetic generation within an LGA run are processed independently, achieving medium-level parallelization. Finally, the low-level strategy consists of the parallel execution of fine-grained tasks that pertain to a single individual, such as rotating the ligand or evaluating the scoring function.

Our implementation (Figure 2) combines the high- and medium-level strategies first. A docking job is composed of $R$ LGA runs ( Run $_{\text {ID }}: 0,1,2, \ldots, R-1$ ), each run processing a population of $P$ individuals $\left(\operatorname{Ind}_{\mathrm{ID}}: 0,1,2, \ldots, P-1\right)$. The execution of such runs, and the processing of their individuals, are controlled by nested loops in the serial implementation, but can be merged into a single loop for optimal parallelism.
By doing so, individuals from different docking runs can be processed simultaneously, each as an OpenCL work-group identified with a $\mathrm{WG}_{\mathrm{ID}}$ obtained as follows:

$$
\mathrm{WG}_{\mathrm{ID}}=\operatorname{Run}_{\mathrm{ID}} * P+\operatorname{Ind}_{\mathrm{ID}}
$$

The entire set of $P * R$ work-groups is distributed by the GPU runtime scheduler over the available $Q$ compute units (CU). Each CU executes a WG associated with a single individual, achieving high- and medium-level parallelization simultaneously. Finally, processing an individual involves fine-grain tasks (genotype generation, ligand rotation, intermolecular and pairwise interactions) that can be assigned to OpenCL work-items, thus achieving low-level parallelization.

Code architecture. The overall workflow of AUTODocKGPU is depicted in Figure 3. This consists of a sequence of functions executed either on the host (H) or on the device (D). After the application inputs are parsed in $\mathrm{H} 1$, the populations of all docking runs are initialized with random values in H2. Then, the OpenCL setup takes place in H3, which comprises the identification and selection of platform and device, as well as the definition of other OpenCL objects such as context and command queues. This process includes the creation of a program object containing all machine-specific instructions to be executed on the device. Since the host is responsible for launching and keeping track of kernel executions, it needs to know how to interact with the kernels. Therefore, in H4, OpenCL kernels (INIT, EVAL, GA, LS) are specified in terms of arguments (variables holding e.g., initial population values, number of genes and ligand atoms, etc.), global size (total number of work-items to be processed by the device), as well as local size (number of work-items to be processed in each work-group).

The first kernel executed is INIT, which calculates the initial score of individuals from all docking runs. The second kernel, EVAL, counts the number of scoring function calls (whose resulting scores reside in device memory) performed by previously-executed kernels. After EVAL executed, control is handed back to the host, which checks whether the LGA termination criteria are met, i.e., if the number of either score evaluations or generations reached their maximum values (default: $2.5 * 10^{6}$ and $27 * 10^{3}$, respectively). The core of the application is the iterative execution of kernels, with each cycle starting with GA, then going through LS, and finishing with EVAL. In particular, the LS kernel executes either the Solis-Wets method, as in the original AUTODock, or the ADADELTA gradient-based method newly added in this work. While the inter-kernel synchronization is controlled entirely on the host via in-order command queues, the transfer of solution data and their scores between kernels occurs by device-side global-memory accesses. Finally, when the LGA termination criteria are met, the final solutions found by the device (and residing in its global memory) are copied back to the host, where results are written to output .dlg files compatible with AuTODockTools (18). 


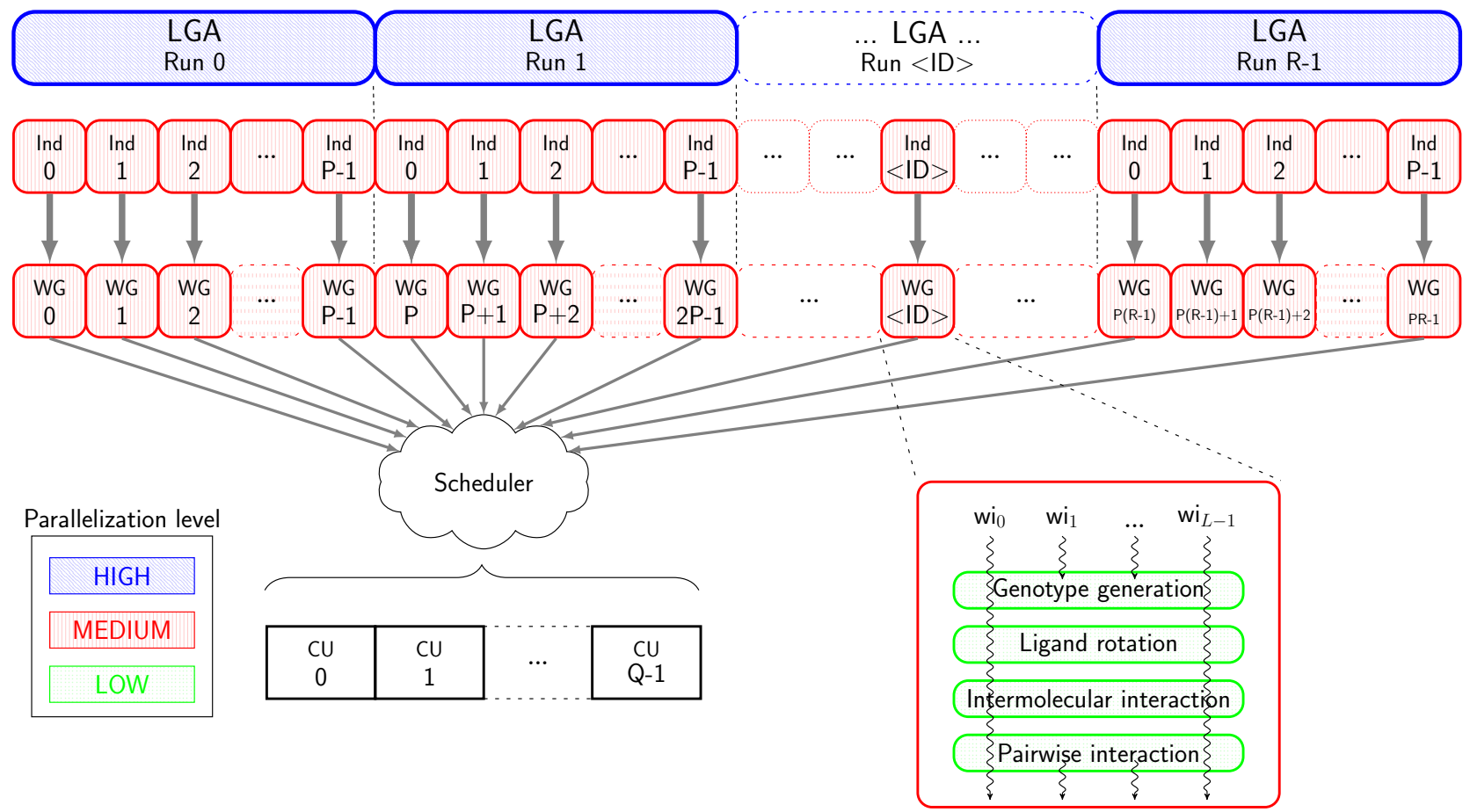

Fig. 2. A population processed by a LGA run ( $\operatorname{Run}_{\mathrm{ID}}$ ) can be decomposed into their individuals, and each individual ( $\left.\operatorname{Ind}_{\mathrm{ID}}\right)$ can be mapped onto a work-group (WG $\mathrm{G}_{\mathrm{ID}}$ ). The entire set of work-groups is distributed by the GPU runtime scheduler over the available $Q$ compute units (CUs). A CU is a multi-threaded hardware unit capable of processing one work-group (composed of $L$ work-items) at a time. The runs, individuals, and fine-grain tasks are colored according to their associated level of parallelism: high (blue), medium (red), and low (green).

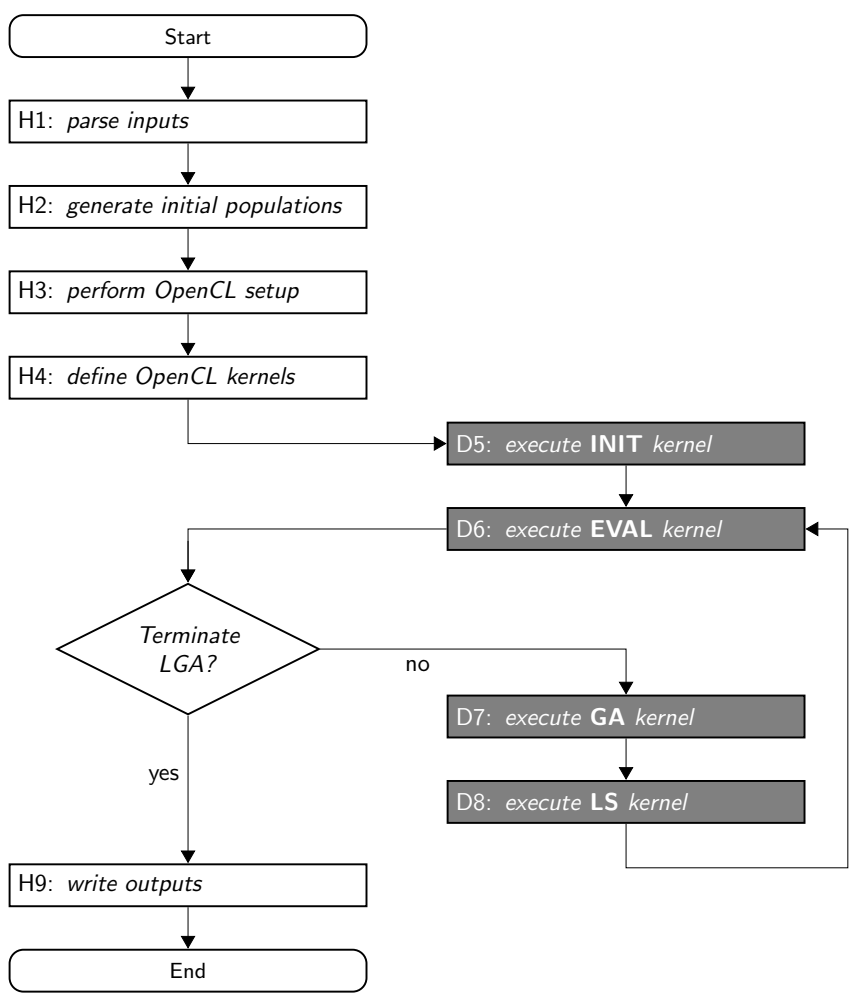

Fig. 3. The overall AUTODOCK-GPU workflow consists of a sequence of host $(\mathrm{H})$ and device (D) functions. Program execution always starts and finishes in host functions (depicted at the left side). Time-consuming functions, i.e., kernels, are executed iteratively on the device (depicted at the right side), while their termination is controlled by the host.
Memory requirements. In order to guarantee that the limited memory capacity (compared to most multi-core CPU servers) of a GPU card does not negatively impact the practical usage of AUTODOCK-GPU, an analysis of the memory size required to hold the processing data is performed. As shown in Table 5, we defined upper limits on AUTODOCKGPU's docking parameters, i.e., the maximum number of elements for the following data: ligand atomic types, ligand atoms, rotatable bonds, pairwise contributors, rotations, population size, docking runs, and grid points. Although by doing this we might constrain the capabilities of AUTODOCKGPU, these limits prevent data allocation beyond the typical memory capacity (few GBs) of most consumer GPU cards.

The first concern is the memory occupied by constant data, which is composed of relatively large look-up tables used in different docking calculations. Table 6 lists all constant arrays utilized when AUTODocK-GPU is configured to run Solis-Wets. These are conveniently grouped into structs (A, $\mathrm{B}, \mathrm{C}, \mathrm{D}, \mathrm{E})$, and passed into GPU memory as OpenCL buffer objects. Depending on the assigned OpenCL memory-space qualifier, a struct can be placed either in the GPU on-board memory (__lobal const), or in the GPU on-chip memory (__constant). Ideally, one would place everything onchip for faster access. However, due to the on-chip capacity limits (in the range of few MB), this is not always possible and consequently, on-board memory must be used as well. The ADADELTA gradient-based search method requires additional space in memory, which is attributed to the structs $(\mathrm{F}, \mathrm{G})$ listed in Table 7. Moreover, grid maps can occupy a large memory region as their size depends exponentially on 
the number of grid points (cubic growth). A maximum limit of 256 grid points would allow users to analyze reasonably large binding regions while keeping the memory space below 1.1 GB. Larger values would require excessive space that cannot be allocated on typical GPU-card memories: e.g., 512 grid points would require more than $8 \mathrm{~GB}$. Then, with the current configuration, the maximum memory space required to store constant arrays is $252 \mathrm{kB}(\mathrm{A}, \ldots, \mathrm{E})+45 \mathrm{kB}(\mathrm{F}, \ldots$, $\mathrm{G})+1,073 \mathrm{MB}($ GRIDS $)=1,074 \mathrm{MB}$, which is possible to be stored on-chip.

Another concern is the storage for variable data, i.e., the information being updated during the entire docking procedure. This data consists of both current and next populations, as well as the scores of their component individuals. As all docking runs are processed in parallel, the maximum memory size required to store current populations $\left(P_{\text {maxsize }}\right)$, and individual scores $\left(E_{\text {maxsize }}\right)$, both expressed in bytes, can be calculated as follows:

$$
\begin{gathered}
P_{\text {maxsize }}=R * P * L_{\text {genotype }} * S_{\text {float }} \\
E_{\text {maxsize }}=R * P * S_{\text {float }}
\end{gathered}
$$

where $R$ and $P$ are respectively the number of docking runs and the population size (both specified by the user), $L_{\text {genotype }}$ is the constant genotype length $(=64)$ in global memory, and $S_{\text {float }}$ is a size of a float or single precision floating-point datatype (4 bytes). For $R$ and $P$ in Equations 3 and 4, we have defined upper limits, too. This means that AuTODocKGPU accepts for $R$ and $P$ any integer value $\leq 1,000$ and $\leq$ 2,048 , respectively. If the user inputs either an invalid or an out-of-range value, then AUTODOCK-GPU outputs a warning message, and then proceeds with execution using default values of $R=1$ and $P=150$. As corner cases, we would have $P_{\text {maxsize }}=1,000 * 2,048 * 64 * 4=524.28 \mathrm{MB}$, and $E_{\text {maxsize }}$ $=1,000 * 2,048 * 4=8.19 \mathrm{MB}$, which together account for 532.48 MB. Considering both - current and next - populations and their scores, their total memory footprint together is $1064.96 \mathrm{MB}$.

Summing up both maximum possible sizes of constant and variable data, the rounded-up memory space required by AutoDock-GPU is less than $2.2 \mathrm{~GB}$, which is lower than the amount typically available on consumer GPU cards, as exemplified in Table 3. Thus, there is no need for computespecialized GPUs with larger memories, which are significantly more expensive.

Main differences compared to AUTODock. The AUTODOCK-GPU implementation involves modifications to the original AUTODock functionality in order to better exploit parallel processing, and the execution performance, without negatively affecting the docking quality. These modifications include the following:

Arithmetic precision. Scoring and search calculations in AUTODOCK are performed using double-precision floating point (64 bits). As previous studies $(23,26,27)$ suggest that performing docking computations with reasonably lower precision does not lead to deterioration in terms of best score and clustering size, we opted to implement those calculations in single-precision floating point (32 bits).

Arrangement of data structures. Data structures were rearranged for better parallel processing of rotation and pairwise interaction. Ligand flexibility can be described by two rotation types. First, a general one that considers the ligand as a rigid body, and a second type due to rotatable bonds for which a tree-like structure is constructed. AuTODock serially traverses the nodes of such flexibility tree in a recursive manner. Although doing so is feasible on OpenCL devices capable of enqueuing kernels independently from the host (a feature known as device-side enqueuing), this would not be portable to devices with more limited language support, i.e., prior to OpenCL 2.0 (28). To tackle this, the recursion is translated into an iterative approach, which is achieved by transforming the flexibility tree into an array-like rotation list. This list is composed of integer-type items (32 bits) with fields detailed in Table 1. Similarly, for the pairwise interaction, instead of having a GPU (likely inefficiently) traversing the tree, the host defines another array-like list, containing intramolecular-contributing atomic pairs.

Selection scheme. Regarding the criterion to choose which individuals will reproduce, the original proportional selection was replaced with binary tournament (default rate: $60 \%$ ). In proportional selection, individuals with better-thanaverage scores receive proportionally more offspring (22). One of its major defficiencies is that if the initial population contains one or two energetically-stronger individuals, then these would dominate the rest, and consequently, would prevent the population from exploring other potentially better solutions by escaping from a local optimum $(29,30)$. On the other hand, in tournament selection, sets of individuals are randomly selected from the entire population. The highest-scoring individual in the set is the tournament winner, and therefore selected for crossover. This scheme also suffers from diversity loss, which happens with large set sizes. Our implementation minimizes this possibility as the minimal tournament set size is chosen (i.e., two, hence the binary denomination). Moreover, the major advantage of tournament selection is the low computational effort, especially if implemented in parallel (30), which according the previous studies $(26,27)$ results in faster executions than those of proportional selection.

Specification of program arguments. AUTODOCK arguments are specified using a docking parameters file (.dpf) containing parameters to control various aspects of a docking job. Here, we replaced the.$d p f$ file with command-line program arguments, making the program more suitable for scripting, which is useful for highly iterative tasks such as virtual screening.

Local search methods. In addition to Solis-Wets, the local search method in the original AUTODOck4, this work additionally newly implements the ADADELTA optimizer 
Table 1. Bit-field description of a 32-bit rotation-list item.

\begin{tabular}{|c|l|}
\hline Bits & Description \\
\hline $7-0$ & ID of atom to be rotated (ATOM \\
\hline $15-8$ & $\begin{array}{l}\text { ID of rotatable bond (ROTBOND } \\
\text { an }) \text { atom with } \text { ATOM }_{\text {ID }} \text { is to be rotated }\end{array}$ \\
\hline 16 & $\begin{array}{l}1: \text { if first rotation of atom with ATOM } \\
\text { 0: otherwise }\end{array}$ \\
\hline 17 & $\begin{array}{l}1: \text { if general rotation, then ROTBOND } \\
\text { I: otherwise }\end{array}$ \\
\hline 18 & $\begin{array}{l}1: \text { if dummy rotation, then no rotation, } \\
0: \text { otherwise }\end{array}$ \\
\hline $31-19$ & Unused \\
\hline
\end{tabular}

based on gradients of the scoring function.

Gradient of the scoring function. The process of docking a ligand is implemented as an optimization problem where different types of ligand motion correspond to variables to be optimized. The first three variables control translation of the ligand in $x, y$, and $z$ directions; the next three variables $\phi, \theta$, and $\alpha$-correspond to rotation of the ligand as a rigid body; and the remaining $\psi$ variables are associated with $N_{\text {rot }}$ rotatable bonds. These variables are also referred to as genes. Collectively, they constitute a genotype, denoted by Omega:

$$
\Omega=x, y, z, \phi, \theta, \alpha, \psi_{1}, \ldots, \psi_{N_{\mathrm{rot}}}
$$

Each genotype corresponds to a different pose of the ligand. The quality of a pose, from the biophysical standpoint, is evaluated by a scoring function $f$ :

$$
f\left(x, y, z, \phi, \theta, \alpha, \psi_{1}, \ldots, \psi_{N_{\text {rot }}}\right)
$$

Here, $f$ is the same scoring function as in Equation 1, but expressed in terms of the optimization variables (genes) instead of interatomic distances and atomic parameters. Docking a ligand consists of finding the genotype $\Omega$ that corresponds to the lowest possible value of the scoring function $f$. Gradientbased optimizers use the gradient $g$ of the scoring function $f$, with respect to the genotype $\Omega$ :

$$
g=\nabla f(\Omega)=\frac{\partial f}{\partial x}, \frac{\partial f}{\partial y}, \frac{\partial f}{\partial z}, \frac{\partial f}{\partial \phi}, \frac{\partial f}{\partial \theta}, \frac{\partial f}{\partial \alpha}, \frac{\partial f}{\partial \psi_{1}}, \ldots, \frac{\partial f}{\partial \psi_{N_{\mathrm{rot}}}},
$$

ADADELTA local search. ADADELTA (19) is a gradientbased optimization algorithm, by which the genotype $\Omega$ is updated at each iteration $t$ :

$$
\Omega_{t+1}=\Omega_{t}+\Delta \Omega_{t}
$$

where $\Delta \Omega_{t}$ is the update vector, which depends not only on the gradient $g$, but also on the history of past gradients and past update vectors:

$$
\Delta \Omega_{t}=-\frac{\sqrt{E\left[\Delta \Omega^{2}\right]_{t-1}+\epsilon}}{\sqrt{E\left[g^{2}\right]_{t}+\epsilon}} g_{t}
$$

where $E\left[\Delta \Omega^{2}\right]$ is a running average of squared updates, and $E\left[g^{2}\right]$ is a running average of squared gradients:

$$
\begin{aligned}
E\left[\Delta \Omega^{2}\right]_{t} & =\rho E\left[\Delta \Omega^{2}\right]_{t-1}+(1-\rho) \Delta \Omega_{t}^{2} \\
E\left[g^{2}\right]_{t} & =\rho E\left[g^{2}\right]_{t-1}+(1-\rho) g_{t}^{2}
\end{aligned}
$$

where $\rho$ and $\epsilon$ are ADADELTA's hyperparameters.

In particular, the constant $\epsilon$ prevents the denominator in Equation 9 from becoming zero if $E\left[g^{2}\right]$ is zero. Furthermore, $\epsilon$ is required to produce non-zero updates in the first iteration $(t=0)$, because the running average of squared updates of the preceeding iteration $E\left[\Delta \Omega^{2}\right]_{-1}$ is assumed to be zero. The running averages $E\left[g^{2}\right]$ and $E\left[\Delta \Omega^{2}\right]$ are vectors of length equal to the number of genetic variables. The update rule is applied to each variable separately, implementing a different learning rate for each dimension. The hyperparameter $\rho$ controls the amount of memory of preceeding iterations for $E\left[g^{2}\right]$ and $E\left[\Delta \Omega^{2}\right]$. Smaller $\rho$ make the running averages more sensitive to the current iteration.

ADADELTA is one of several first-order local optimization algorithms. Ideally, we would have implemented a QuasiNewton method, such as BFGS (31), but we opted for a firstorder method because it is simpler to be integrated in our highly-parallel OpenCL architecture. Examples of other popular first-order methods are Adam (32), and FIRE (33). All these algorithms have hyperparameters that influence the calculation of the update vector. We chose ADADELTA because it has only two hyperparameters, $\rho$ and $\epsilon$, making it simpler to optimize the algorithm for docking, while Adam and FIRE have four and five hyperparameters, respectively. After evaluating ADADELTA using various combinations of $\rho$ and $\epsilon$ we set $\rho=0.8$ and $\epsilon=0.01$, because these values provided the best search performance (see Figure 6 and associated discussion).

Dataset. A dataset of 140 protein-ligand complexes was used to compare our OpenCL implementation to AuToDock4. Our dataset is mainly sourced from two wellestablished sets for assessing docking methodologies for drug discovery purposes: the Astex diversity set (34) and CASF2013 (35). We included all 85 entries from the Astex diversity set, in which most ligands have up to 10 rotatable bonds. With regard to the CASF-2013 set, we included 35 complexes, enriching our set with ligands in the range of 11 20 rotatable bonds. Furthermore, we included 20 additional complexes selected from the Protein Data Bank (36) covering a large range of rotatable bonds (up to 32) and displaying both wide and narrow binding pockets.

Identification of global minima. We identified global minima for the complexes in our dataset by analyzing the distribution of scores produced by LGA runs (Figure 4). Increasing the number of evaluations shifts the distribution of scores towards more negative values (i.e., better scores). For most complexes, we observed convergence of the distributions towards a lower bound: once that lower bound is reached, increasing the number of evaluations does not change the lower bound, but increases the number of LGA runs that actually found the lower bound score. The upper panel of Figure 4 shows an example of convergence towards a lower bound. Based on this observation, we devised a criterion to systematically identify global optima, which is explained as follows: for a protein-ligand complex, we consider the scores returned 
by LGA runs with up to 8,192,000 evaluations. A total of 4 docking replicates are performed, where each replicate starts with a different conformation and orientation of the input ligand. From each replicate, we retrieve the 5 top scores (out of 100 LGA runs), creating a subset of 20 scores. This procedure is repeated for 4 docking replicates with 100 LGA runs each, executed up to 4,096,000 evaluations. The best score from each replicate is added to the subset of scores, increasing its size from 20 to 24. If the difference between the best and worst scores within the subset (of 24 scores) is less or equal to $0.1 \mathrm{kcal} / \mathrm{mol}$, the best score is considered to be the global minimum. While there is no theoretical guarantee that an even lower minimum does not exist, we did not observe a single case in which the distribution of scores initially converged to an intermediate value, and then escaped to an even lower score after further evaluations. This suggests that the distribution of scores is not easily trapped in local minima. Moreover, varying local-search rate and local-search method results in convergence to identical global minima: the largest difference in global minima found from different dockings was just $0.23 \mathrm{kcal} / \mathrm{mol}$.

Technical details. All calculations reported in this work employ a maximum of 300 local-search iterations. The maximum number of LGA generations is set to 99,999 to guarantee that docking does not stop before the maximum number of score evaluations is reached.

Ligand and receptor input files were prepared with OpenBabel-2.4.1 (37), after removing all water molecules and metal atoms for which AUTODOCK4 misses atom types.

Regarding software support, the latest AUTODOCK4.2.6 was used as a single-threaded CPU baseline. For GPUs, OpenCL drivers provided within the CUDA 10.0 and AMDAPPSDK 3.0 packages were used. For evaluating the portability of OpenCL to multicore CPU machines, we employed the Intel SDK-2017 driver.

Measuring the evaluation rate. Docking a ligand involves a large number of score evaluations, often in the order of millions. The time spent per evaluation is one of the factors determining docking runtime. To calculate the evaluation rate, we run dockings using a varying number of evaluations, and fit a linear model defining the docking runtime $y$ as a function of the number of evaluations $x$ :

$$
y=a x+b
$$

where $b$ is the intercept with the y-axis and $a$ is the slope.

The slope $a$ is the rate at which score evaluations are performed, which we chose to express in microseconds $(\mu s)$ per evaluation. Dockings were performed from 32,000 to 2,048,000 evaluations, in exponential increments of 2-fold, providing 7 datapoints to fit the linear model from which the evaluation rate is determined. Four replicates were performed for each docking, and the minimum runtime was considered. Identical results would be obtained if the average runtime were considered instead of the minimum (evaluation rates increased on average by only $1 \%$ ). Evaluation rates for which the coefficient of determination $R^{2}$ is less than 0.99 were excluded. In the Results and Discussion section, we report the evaluation rate for all 140 complexes in our dataset, running AUTODOCK-GPU on different accelerators, and using either Solis-Wets or ADADELTA as local-search methods.

\section{RESULTS}

Validating the scoring function implementation. Docked poses from AUTODOCK-GPU .dlg output files were re-scored using AUTODocK4.2.6 (keyword $e p d b$ ). A total of 1015 ligand poses from 10 complexes were considered in this analysis. We observe that for docked poses in the favorable range (no clashes), the differences are in the order of $0.01 \mathrm{kcal} / \mathrm{mol}$ for grid-interpolated energies, and $0.02 \mathrm{kcal} / \mathrm{mol}$ for ligand-ligand (pairwise) interactions. In the case of unfavorable energies, such as when clashes occur, larger differences were observed. These differences are irrelevant for drug-design applications, because the interest is in the most favorable poses of each ligand, whereas high-energy (unfavorable) poses are discarded.

Score differences between AUTODock4 and AutoDocKGPU are mainly attributed to the following two reasons. First, scoring and search computations in AUTODOCK-GPU are performed with single-precision arithmetic, which might cause a loss of precision in comparison to double-precision floats used in AUTODocK4. Second, for quantifying ligandligand interactions, AUTODOCK4 interpolates values from look-up tables, while AUTODocK-GPU evaluates the analytical form of the scoring function $f$ (Equation 1). This implementation discrepancy can cause larger score differences - in the order of 1 or more $\mathrm{kcal} / \mathrm{mol}$ - because some of the scoring terms have steep energy profiles for short interatomic distances. Despite the fact that score differences between AutoDock4 and AUTODOCK-GPU are insignificant for well optimized ligand poses without steric clashes, users should be aware of significant discrepancies in high-energy poses arising primarily from the interpolation of ligandligand terms in AuTODock4. In this regard, AUTODocKGPU is a more accurate implementation of the scoring function.

Algorithmic performance. In the field of global optimization, computational effort is often expressed as the number of fitness (or score) evaluations (38). More efficient algorithms spend fewer evaluations to achieve a certain level of performance.

Despite that the number of score evaluations may not correlate with runtime, using it for assessing algorithms, such as Solis-Wets and ADADELTA, is advantageous. The reason is that, in contrast to runtime, the number of score evaluations allows the assessment of algorithmic performance independently from code optimization, choice of compiler, and hardware specifications. 


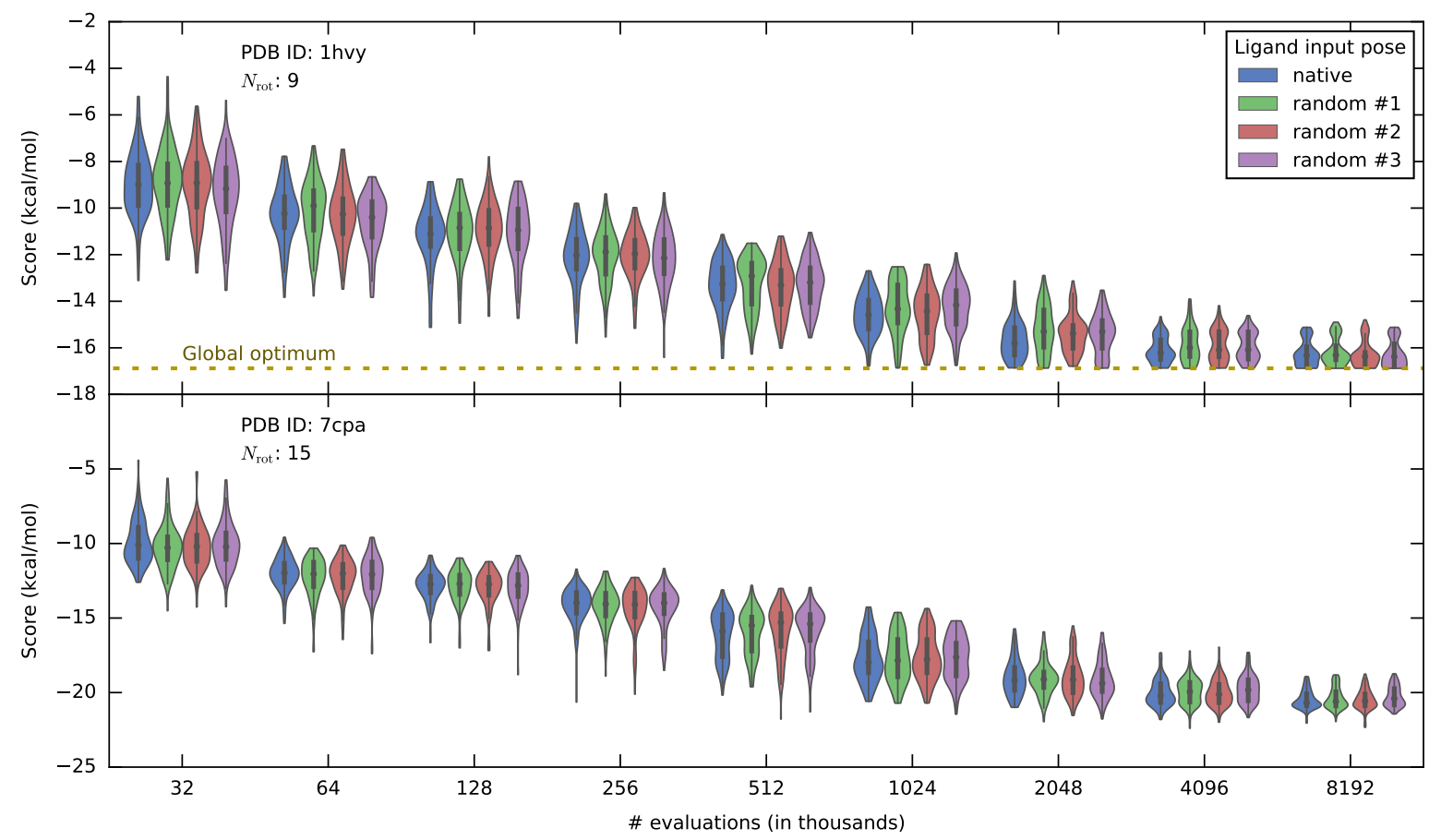

Fig. 4. Distribution of scores returned by LGA runs with increasing number of evaluations. Each violin plot represents scores from 100 LGA runs, and the color is associated with the input conformation and orientation of the ligand. The global minimum was identified for the protein-ligand complex represented in the upper panel (PDB ID: 1 hvy), but not for the complex in the bottom panel (PDB ID: 7cpa) because the distribution of scores did not converge towards a lower bound. The local-search method used is ADADELTA, and the local-search rate is $100 \%$.

Success rate of LGA runs. Our assessment of search performance starts by determining if individual LGA runs are successful or not. We use two criteria to define success, one based on the docking score, and another based on the root mean square deviation (RMSD) from the native pose determined by X-ray crystallography.

According to the score criterion, an LGA run is successful if the returned pose has a score within $1.0 \mathrm{kcal} / \mathrm{mol}$ from the best possible score for a given protein-ligand complex, i.e., the global minimum. The protocol for finding global minima in our dataset is described in the Methodology section. We found global minima for 105 out of 140 complexes, while for the rest that was not possible due to the presence of a large number of rotatable bonds, particularly for ligands containing more than 19 of these.

According to the RMSD criterion, an LGA run is successful if the returned pose is within $2 \AA$ from the native pose. We note that the job of search algorithms is to find the global minimum of the scoring function, and there is no guarantee that the global minimum corresponds to a pose that is within $2 \AA$ RMSD from the native pose. This happens because scoring functions are not perfect, and sometimes give better scores to incorrect poses. Since our goal is to evaluate search performance, we need to guarantee that the optimization target of the search algorithm (i.e., the global minimum of the scoring function) corresponds to a solution that is deemed correct by the success criterion. Therefore, we only use the RMSD criterion when the global minimum of the scoring function is below $2 \AA$ RMSD of the actual physical pose ( 78 out 140 complexes in our dataset).

Since the LGA algorithm is stochastic, we employ a statistical approach involving a relatively large number of LGA runs. Specifically, we perform four replicates of 100 LGA runs, each replicate starting with the ligand in a different orientation and conformation: one of the four inputs is the native pose, whereas the other three are a randomized pose each (AUTODOCK VINA (8) was used for randomization). Using this data, we calculate the fraction of successful LGA runs.

The fraction of successful LGA runs increases with the number of evaluations, and its corresponding success-rate curve typically follows a sigmoidal profile (Figure 5). An insufficient number of evaluations results in a low (possibly zero) probability of finding either the native pose or the global minimum. After a certain number of evaluations, the success probability asymptotically approaches $100 \%$. Increasing the number of evaluations beyond the higher inflexion point provides only relatively small increases $(\sim 10 \%)$ in the probability of success.

Halfway number of evaluations $\left(E_{50}\right)$. From success probability curves (Figure 5), we estimate the number of evaluations required to achieve a $50 \%$ probability of success. We refer to this number as halfway number of evaluations, and abbreviate it as $E_{50}$.

The values of $E_{50}$ depend on both the protein-ligand complexity, as well as the algorithmic efficiency of search methods. If the global minimum (or native pose) of a given protein-ligand complex is easy to find, high-success proba- 


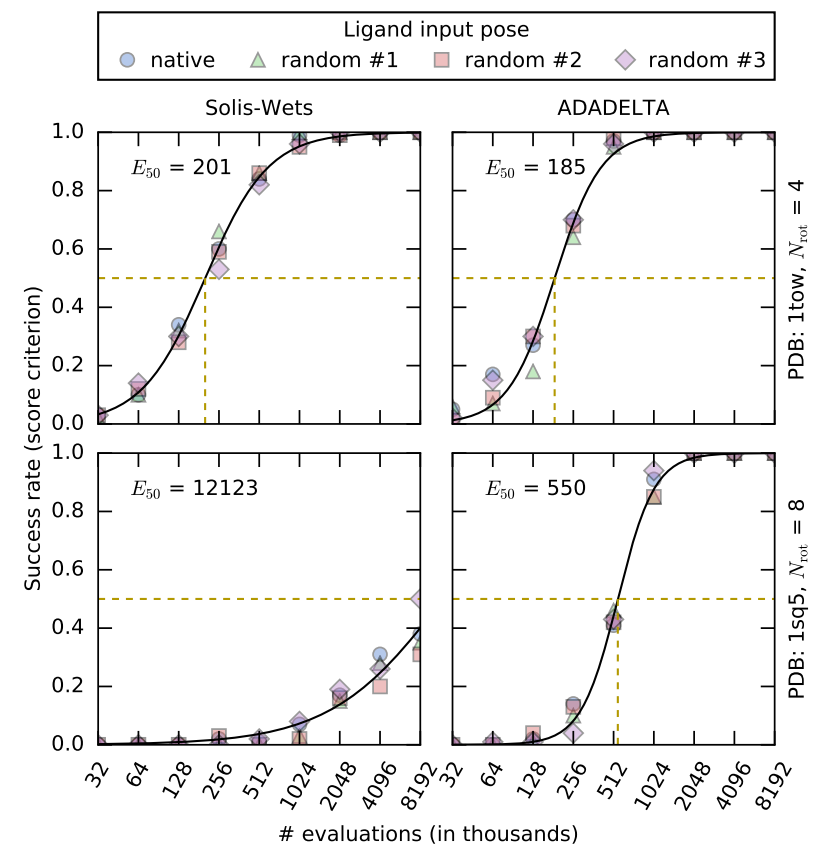

Fig. 5. Fraction of successful LGA runs as a function of the number of score evaluations, using Solis-Wets and ADADELTA as local-search methods. The upper plots correspond to an easy search problem with only four rotatable bonds (PDB ID: 1tow), while the bottom plots correspond to a moderately difficult ligand with eight rotatable bonds (PDB ID: 1sq5). According to the score criterion, an LGA run is successful if it reports a pose within $1.0 \mathrm{kcal} / \mathrm{mol}$ from the global optimum. The black line is a fitted sigmoid curve (Equation 13) that estimates $E_{50}$, which is the number of evaluations at which $50 \%$ of LGA runs are successful. The dashed orange lines are a visual representation of $E_{50}$ values.

bilites are achieved with a relatively small number of evaluations. Analogously, lower $E_{50}$ numbers indicate more efficient search algorithms, because the halfway performance is achieved with less computational effort, i.e., fewer score evaluations. This is illustrated in Figure 5, where the top and bottom panels correspond to an easy and (moderately) difficult case, respectively. For example, considering the successrate curves for the moderately difficult complex (PDB ID: 1sq5) in Figure 5, it can be inferred that ADADELTA outperforms Solis-Wets because it requires $\sim 20 \mathrm{x}$ fewer evaluations to achieve $50 \%$ of success rate. In fact, for this complex, using Solis-Wets - with 8 million evaluations per LGA run - results in success rates between $30 \%$ and $50 \%$, while ADADELTA approaches $100 \%$ of success with only 2 million evaluations.

In order to calculate $E_{50}$ values, we fit the following sigmoid function to the success-rate curves depicted in Figure 5:

$$
\text { Fraction of successful LGA runs }=\frac{1}{1+e^{-\beta\left(x-E_{50}\right)}}
$$

where $x$ is the number of score evaluations, while $\beta$ and $E_{50}$ are variable parameters optimized during sigmoid fitting.

Since $E_{50}$ values describe search performance by a single number, we use it to assess search performance on a large number of protein-ligand complexes, by plotting $E_{50}$ values as a function of the number of ligand rotatable bonds $N_{\text {rot }}$. Search difficulty increases with $N_{\text {rot }}$, because each rotatable bond adds one dimension to the optimization problem. In the following sections, we use $E_{50}$ values to guide the optimization of ADADELTA hyperparameters, and to demonstrate the superiority of ADADELTA over Solis-Wets for large $N_{\text {rot }}$.

Tuning ADADELTA hyperparameters. As previously mentioned, ADADELTA has two hyperparameters: $\rho$ and $\epsilon$. In an initial stage of this work, we manually tuned these hyperparameters to yield low scores in as few evaluations as possible, using a small number of protein-ligand complexes, and visualizing the progression of scores over a few hundred iterations. Eventually, we settled on $\rho=0.8$, and $\epsilon=0.01$, but noted afterwards that such $\rho$ was smaller than the smallest $\rho$ $(=0.9)$ tested in the original ADADELTA publication (19).

To validate our initial choice of hyperparameters, we used a subset of 19 protein-ligand complexes from our dataset (for which global minima were identified), and tested six combinations of $\rho(0.8,0.9,0.95)$, and $\epsilon(0.01,0.0001)$ values. Larger values for $\epsilon$ result in larger initial steps, because in the first ADADELTA iteration $(t=0)$ the running average of squared updates of the preceeding iteration $E\left[\Delta \Omega^{2}\right]_{-1}$ is assumed to be zero, so the magnitude of the update is scaled by $\sqrt{\epsilon}$ (Equation 9). Smaller values for $\rho$ reduce the impact of preceeding iterations, making the optimization more sensitive to the gradient of the current iteration (Equation 11).

Search performance was measured by $E_{50}$ values, calculated according to the score criterion (LGA is successful if reported pose is within $1.0 \mathrm{kcal} / \mathrm{mol}$ from the global optimum). By plotting $E_{50}$ as a function of $N_{\text {rot }}$ (Figure 6), we observed that our initial hyperparameter setup $(\rho=0.8$, and $\epsilon=0.01)$ yields the lowest $E_{50}$ values among the tested hyperparameter combinations. Decreasing $\epsilon$ from 0.01 to 0.0001 results in larger $E_{50}$ (i.e., lower performance), while increasing $\rho$ results in very small performance gains. Fitting an exponential equation of the form $E_{50}=2^{a * N_{\text {rot }}+b}$ (green dashed lines in Figure 6) helps in quantifying performance: the lower the regression coefficients ( $a$ and $b$ ), the smaller $E_{50}$, and thus the higher performance. This regression analysis confirms the visual interpretation of $E_{50}$ plots, as $\rho=0.8$, and $\epsilon=0.01$ result in the lowest $a$ and $b$, and thus the smallest number of iterations to reach a $50 \%$ probability of computing the optimum solution.

\section{Algorithmic performance of ADADELTA and Solis-Wets. The algorithmic performances of ADADELTA} and Solis-Wets were compared by calculating their $E_{50}$ values for all protein-ligand complexes in our dataset. Moreover, for both LS methods, $E_{50}$ was calculated as a function of $N_{\text {rot }}$, as well as for three different rates of performing local search on a genetic population (LS rate): $6 \%, 25 \%$, and 100\% (Figure 7).

In general, both LS methods require more score evaluations to achieve $E_{50}$ if the ligand has a larger number of rotatable bonds (Figure 7). Interestingly, when using Solis-Wets, $E_{50}$ values increase more steeply with $N_{\text {rot }}$ than when using ADADELTA. This occurs for $E_{50}$ values calculated based on 


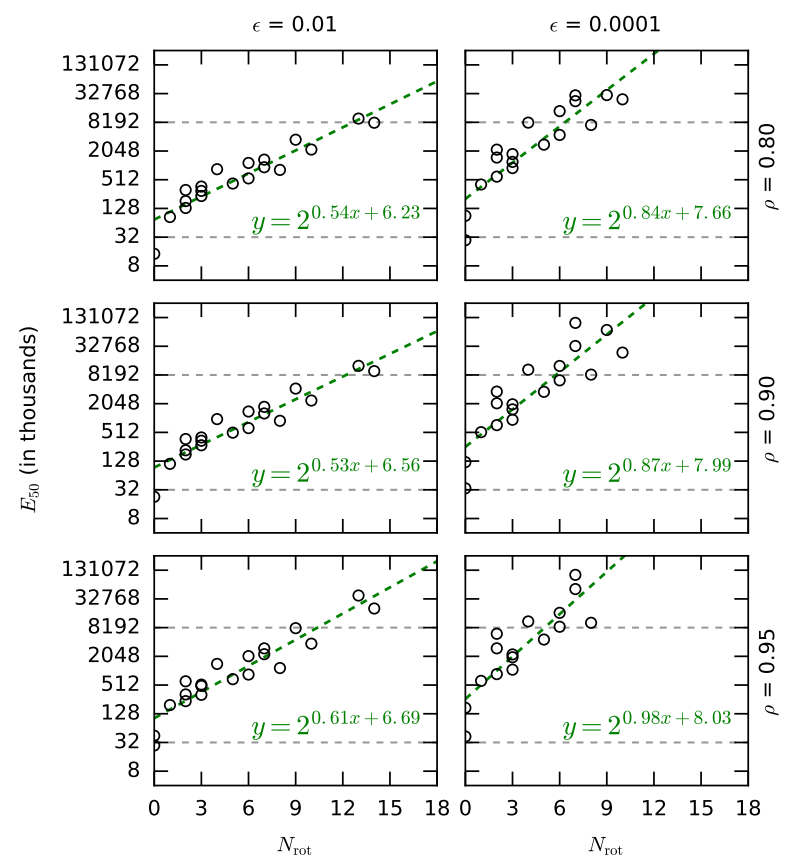

Fig. 6. Dependency of $E_{50}$ on the number of rotatable bonds $N_{\text {rot }}$ for six combinations of ADADELTA hyperparameters $\rho$ and $\epsilon$. The score criterion was used to calculate $E_{50}$ values. The green dashed lines represents the fitted equation (also in green). A total of 19 protein-ligand complexes were used, but it was not always possible to fit $E_{50}$ values, so there are less than 19 data-points in some plots. Local-search rate is $100 \%$, and the maximum number of local-search iterations is 300 .

both the score and RMSD as criteria for LGA success. When docking ligands with many rotatable bonds, ADADELTA reaches a 50\% success rate with fewer evaluations than SolisWets. Notably, using Solis-Wets, we could not fit sigmoid curves (Equation 13) for any ligand containing 12 or more rotatable bonds because the LGA success rates were too low. On the other hand, using ADADELTA resulted in $E_{50}$ below 8 million for some complexes with more than 15 rotatable bonds.

Analysis of regression lines associated with the equation $E_{50}=2^{a * N_{\mathrm{rot}}+b}$ reveals that increasing the LS rate results in lower coefficients $a$ and $b$, and hence lower average $E_{50}$ values. We argue that the $a$ coefficient is more important than $b$ because it dictates how $E_{50}$ scales with the number of rotatable bonds. The influence of LS rate is more pronounced for ADADELTA than for Solis-Wets, with noticeable improvements in $E_{50}$ values after increasing LS rate from $6 \%$ to $25 \%$. Moreover, there is virtually no difference in performance when using either $25 \%$ or $100 \%$ LS rate, but using $100 \%$ is recommended because the corresponding $a$ coefficients are slightly lower.

Let us compare the algorithmic performance of Solis-Wets and ADADELTA by using their $E_{50}$ values, calculated according to the score criterion, and $100 \%$ LS rate. The regression model predicts an $E_{50}$ value of $\sim 2^{0.99 N_{\text {rot }}+5.50}$ for Solis-Wets, while for ADADELTA an $E_{50}$ value of $\sim$ $2^{0.40 N_{\text {rot }}+6.94}$ is predicted. For a ligand with zero rotatable bonds, the Solis-Wets $E_{50}$ would be $\sim 45,000$ evaluations, whereas the ADADELTA $E_{50}$ would be $\sim 123,000$.
Therefore, ADADELTA needs $\sim 3 \mathrm{x}$ more evaluations than Solis-Wets. If the number of rotatable bonds is increased to 12 , Solis-Wets would need $\sim 170,000,000$ evaluations to reach a 50\% success rate, while ADADELTA requires only $3,400,000$ (a 50x reduction). Assuming these equations are still valid for 20 rotatable bonds, this factor would increase to $\sim 1300 \mathrm{x}$. In other words, ADADELTA would need $\sim 30$ million evaluations, while Solis-Wets is predicted to require $\sim 40$ billion instead.

The agreement of $E_{50}$ values calculated using both the score and RMSD criteria gives us more confidence in our analysis. Before using the RMSD criterion, we hypothesized that the role of ADADELTA was merely in removing clashes from poses that were already very close to the X-ray pose, and that Solis-Wets was just as good at producing poses within $2 \AA$ RMSD from the X-ray, even though with less favorable energies. The RMSD criterion for LGA success invalidates this hypothesis, and shows that ADADELTA helps the LGA to find the native pose.

To better determine the number of rotatable bonds beyond which ADADELTA outperforms Solis-Wets, we plotted Solis-Wets $E_{50}$ values against those of ADADELTA (Figure 8), both obtained using an LS rate of $100 \%$. Ligands with three or fewer rotatable bonds (light blue circles) are typically below the equality line, which means that SolisWets has lower $E_{50}$ values, and hence a higher algorithmic performance. On the other hand, and according to the score criterion, ligands with four or more rotatable bonds have lower $E_{50}$ values using ADADELTA. Interestingly, according to the RMSD criterion, some ligands with $N_{\text {rot }}$ between four and nine have lower $E_{50}$ with Solis-Wets. Nevertheless, the majority of ligands with four or more rotatable bonds have lower $E_{50}$ values with ADADELTA, according to either the score or RMSD criteria. This analysis considers only complexes for which $E_{50}$ values could be calculated for both Solis-Wets and ADADELTA, so the maximum number of rotatable bonds is 11 (Solis-Wets has very low success rates for ligands with 12 or more rotatable bonds, and $E_{50}$ values could not be estimated). Based on these results, we conclude that Solis-Wets has better algorithmic performance for ligands with fewer than three rotatable bonds, while ADADELTA has better algorithmic performance for ligands with four or more rotatable bonds.

Evaluation rate performance. The runtime of docking depends mostly on the number of evaluations, often reaching hundreds of millions. For example, performing 100 LGA runs with 2.5 million evaluations each results in 250 million evaluations being executed in a single docking. For this reason, the evaluation rate is a useful indicator of docking performance. In this section, we evaluate some speedups attained by GPU accelerators based on the evaluation rate the time spent per score evaluation - expressed in microseconds $(\mu \mathrm{s})$ per evaluation.

In Figure 9, we depict the evaluation rate attained when using our full dataset, and executing AUTODocK-GPU on differ- 
Solis-Wets
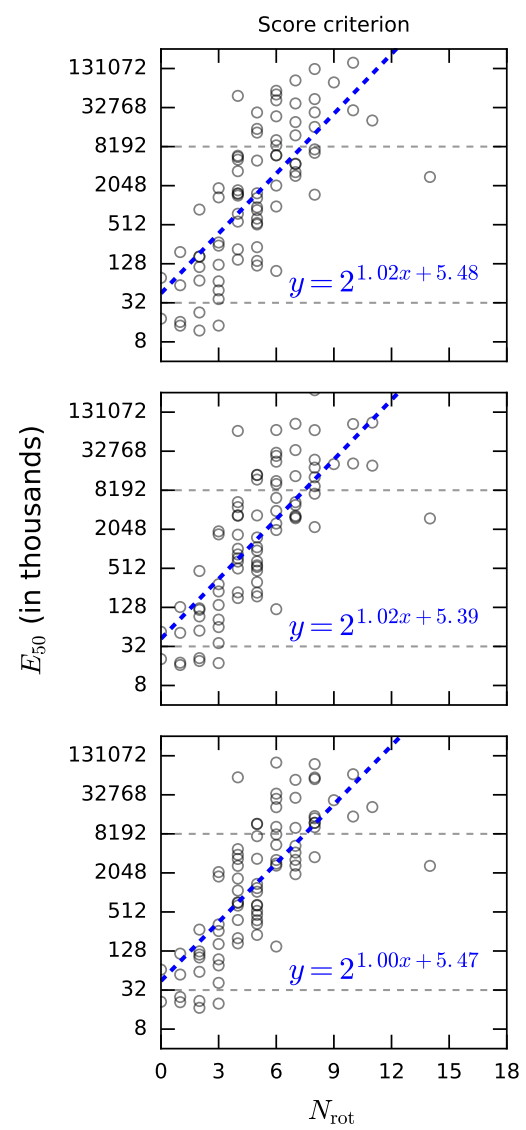
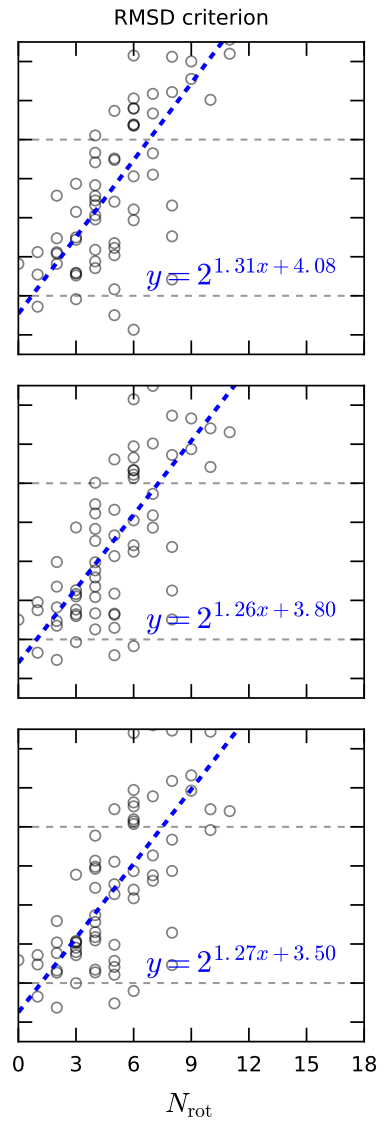
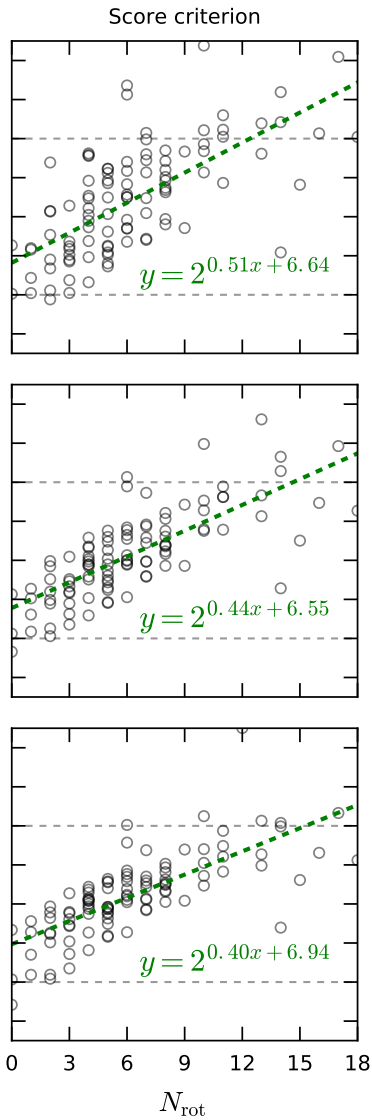

ADADELTA
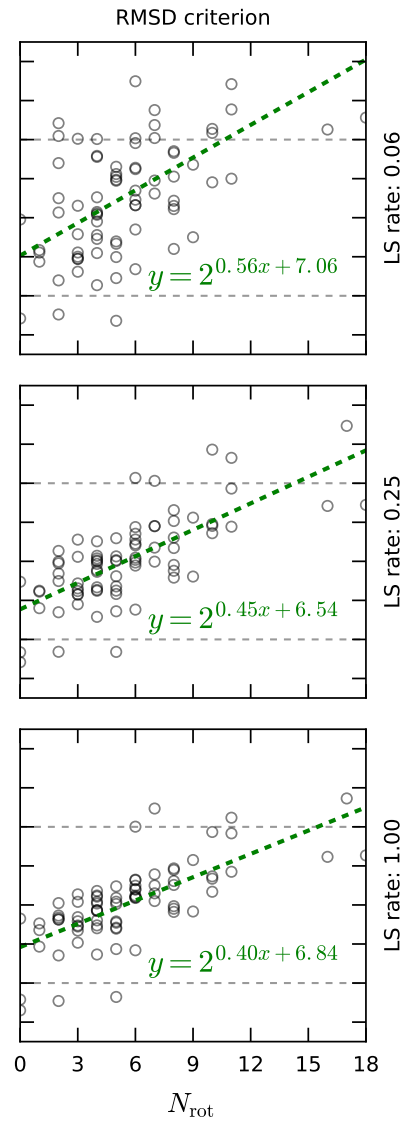

Fig. 7. Dependency of $E_{50}$ on the number of ligand rotatable bonds $N_{\text {rot }}$ for Solis-Wets and ADADELTA local-search methods. The LS rate is $6 \%$ in the top row, $25 \%$ in the middle row, and $100 \%$ in the bottom row.

ent GPUs (listed in Table 3), as well as AUTODocK4 on a single CPU core. Evaluation rate speedups for each complex in our dataset were calculated by dividing the evaluation rate of AutoDock4 by that of AUTODock-GPU. To establish a fair baseline, AUTODOCK4 was run on a recent compute instance (c5.18xlarge) - provided by Amazon Web Services (AWS) - that features an Intel Xeon Platinum 8124M CPU @ 3.00GHz (Table 4).

AUTODocK-GPU achieves the fastest evaluation rate on a GTX 1080 Ti GPU using Solis-Wets, spending between $0.007-0.28 \mu$ s per evaluation, depending on the proteinligand complex. In contrast, AUTODOCK4 yields the lowest evaluation rate, ranging between 1.25 - $54.7 \mu$ s per evaluation.

In all platforms, more ligand atoms lead to slower calculations. For Solis-Wets, the time per evaluation increases in an almost linear fashion with the number of ligand atoms $\left(N_{\text {atom }}\right)$. For ADADELTA, the time per evaluation increases in a more quadratic fashion. In consequence, ADADELTA becomes slower in comparison to Solis-Wets for large $N_{\text {atom }}$ values. To provide an estimate, ADADELTA is about $4 \mathrm{x}$ as slow as Solis-Wets for ligands with $\sim 20$ atoms, and about $16 \mathrm{x}$ slower for ligands with $\sim 100$ atoms.
Table 2. Evaluation rate ( $\mu$ s/evaluation) for different GPU platforms and varying number of ligand atoms. Evaluation rates reported here are interpolated by polynomial fitting as shown in Figure 9.

\begin{tabular}{|l|rrr|rrr|}
\multicolumn{1}{c|}{} & \multicolumn{3}{c|}{ Evaluation rate $(\mu$ s / evaluation) } \\
\cline { 2 - 8 } \multicolumn{1}{c|}{} & \multicolumn{3}{c|}{ Solis-Wets } & \multicolumn{3}{c|}{ ADADELTA } \\
\hline \# Ligand atoms & 20 & 40 & 80 & 20 & 40 & \multicolumn{1}{c|}{80} \\
\hline M2000 & 0.22 & 0.49 & 1.21 & 0.39 & 2.76 & 16.11 \\
GTX 980 & 0.05 & 0.13 & 0.37 & 0.21 & 1.03 & 5.62 \\
Vega 56 & 0.04 & 0.10 & 0.31 & 0.16 & 0.77 & 4.08 \\
GTX 1080 Ti & 0.03 & 0.06 & 0.17 & 0.06 & 0.44 & 2.40 \\
\hline AUTODOCK4 & 3.66 & 10.64 & 34.58 & & & \\
\hline
\end{tabular}

Overall, the evaluation rate speedup achieved by each GPU depends on the hardware specifications (summarized in Table 3), with more powerful GPUs attaining larger speedups. Furthermore, evaluation rate speedups depend not only on the accelerator platform, but also on the choice of LS method and the number of ligand atoms (Figure 10). For dockings using Solis-Wets, evaluation rate speedups increase with the number of ligand atoms, while the opposite behavior occurs for ADADELTA. In the case of very large ligands ( $~ 100$ atoms), we observed evaluation rate speedups of ADADELTA being lower than 10x on a Vega 56 GPU. 
Table 3. Comparison of selected hardware characteristics of the evaluated GPU-based accelerator cards.

\begin{tabular}{|c|c|c|c|c|c|c|c|}
\hline \multirow[t]{2}{*}{$\begin{array}{l}\text { GPU-based } \\
\text { Accelerator } \\
\text { Card }\end{array}$} & \multirow{2}{*}{$\begin{array}{l}\text { Peak } \\
\text { Memory } \\
\text { Bandwidth } \\
(\text { GB/s) }\end{array}$} & \multicolumn{2}{|c|}{$\begin{array}{l}\text { Peak Compute } \\
\text { Performance } \\
\text { (GFLOPS/s) }\end{array}$} & \multirow[t]{2}{*}{$\begin{array}{l}\text { On-board } \\
\text { Memory } \\
\text { (GB) }\end{array}$} & \multirow[t]{2}{*}{$\begin{array}{c}\text { Release } \\
\text { Year }\end{array}$} & \multicolumn{2}{|c|}{$\begin{array}{c}\text { Geometric } \\
\text { Average } \\
\text { Evaluation Rate Speedup }\end{array}$} \\
\hline & & FP32 & FP64 & & & Solis-Wets & ADADELTA \\
\hline M2000 & 105.8 & 1786 & 55.82 & 4 & 2016 & 19.6 & 5.4 \\
\hline GTX 980 & 224.4 & 4981 & 155.6 & 4 & 2014 & 75.4 & 14.9 \\
\hline Vega 56 & 409.6 & 10566 & 660.4 & 8 & 2017 & 94.4 & 17.3 \\
\hline GTX $1080 \mathrm{Ti}$ & 484.4 & 11340 & 354.4 & 11 & 2017 & 157.8 & 36.0 \\
\hline
\end{tabular}

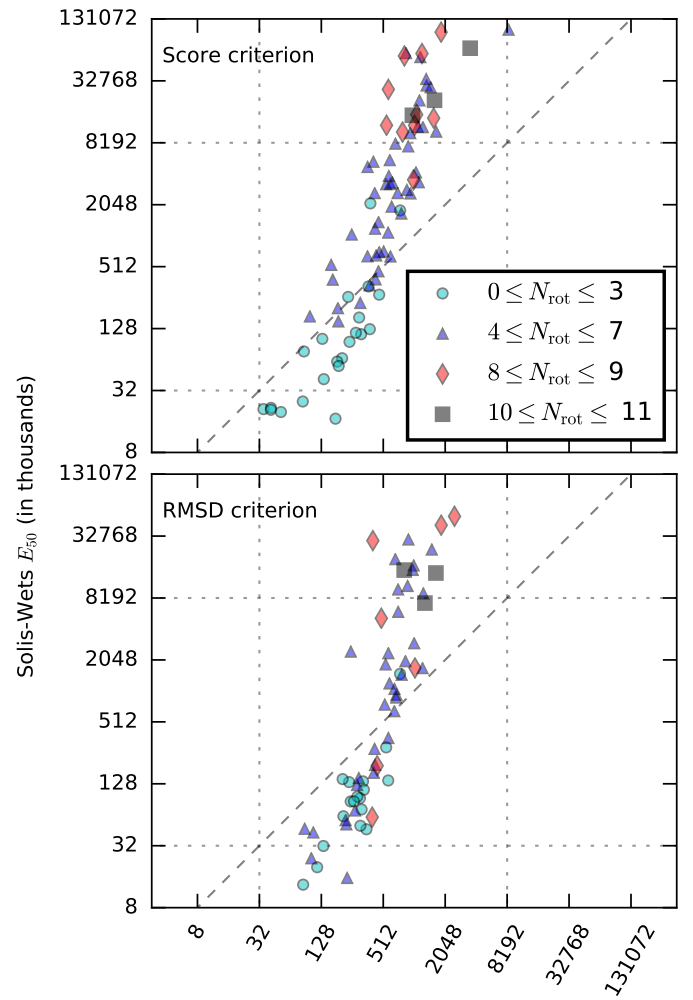

ADADELTA $E_{50}$ (in thousands)

Fig. 8. Direct comparison of $E_{50}$ values between ADADELTA and Solis-Wets using $100 \%$ LS rate.

\section{Balancing algorithmic and evaluation rate} performance. In our previous analysis, we estimated algorithmic improvements of ADADELTA over Solis-Wets to be in the order of 50x (for ligands with 12 or more rotatable bonds), as well as faster executions of Solis-Wets compared to ADADELTA in the order of 16x (for ligands containing $\sim 100$ atoms). Thus, for ligands with 12 or more rotatable bonds, ADADELTA is the obvious choice, because the reduction in the number of score evaluations outweighs the faster evaluation rate of Solis-Wets.

However, the choice between these LS methods is less obvious for ligands with fewer than 12 rotatable bonds. To clarify this, we calculated the time required to perform $E_{50}$ evaluations with each method. This time-to- $E_{50}$ is the product of $E_{50}$ by the corresponding evaluation rate ( $\mu$ s / evaluation) for each protein-ligand complex. By plotting the resulting time-to- $E_{50}$ values of Solis-Wets against those of ADADELTA (Figure 11), we compare both LS methods in

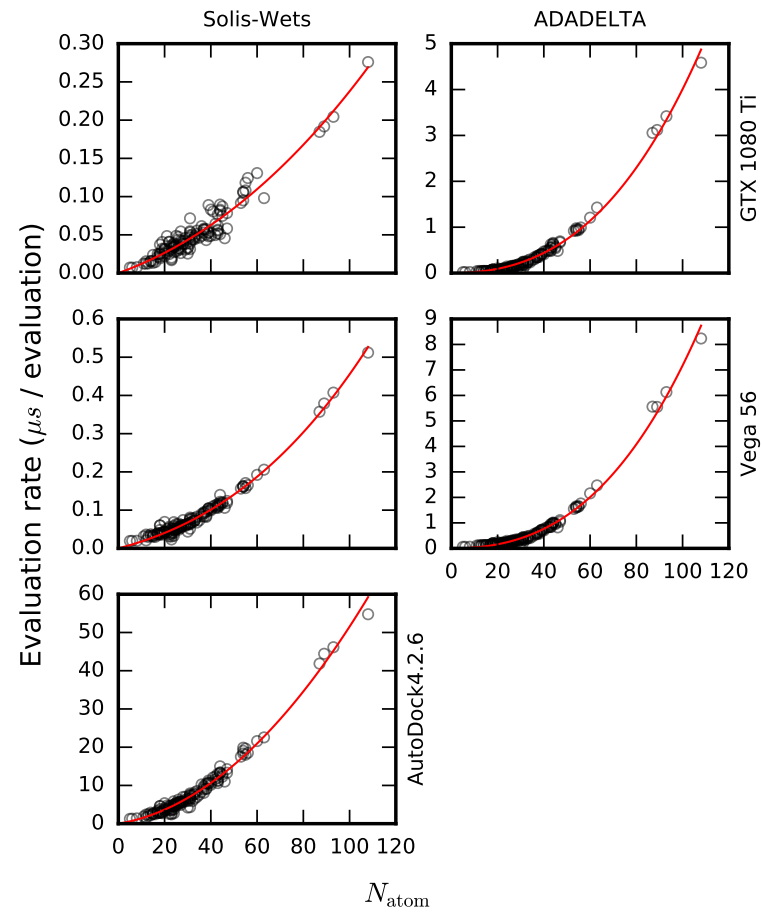

Fig. 9. Evaluation rate of AUTODOCK-GPU and AUTODock4. Note that each subplot has a different scale for the $y$-axis. The red lines are third-degree polynomial fits. These fits were used to interpolate the evaluation rate values reported in Table 2 .

terms of time efficiency for reaching a $50 \%$ success rate on a single LGA run. While ADADELTA is successful in fewer evaluations than Solis-Wets for ligands with four or more rotatable bonds, the faster evaluation rate of Solis-Wets compensates for the larger number of evaluations. As a result, ADADELTA is more time-efficient for ligands with eight or more rotatable bonds, while Solis-Wets is more time-efficient for ligands with seven or fewer rotatable bonds. These numbers were calculated using evaluation rates obtained with the Vega 56 GPU, which has an intermediate performance within the range of GPU cards we tested. While results may be slightly different on other GPU cards, this analysis provides an estimate of the time-efficiency of each LS method.

Additionally, we note that in the current version of AUTODOCK-GPU, the number of evaluations is predetermined at the start of docking by a (user) commandline option. Thus, the time-to-completion of docking is not strictly related to the time-to- $E_{50}$ described above. However, in the future, self-tunning stopping criteria may 


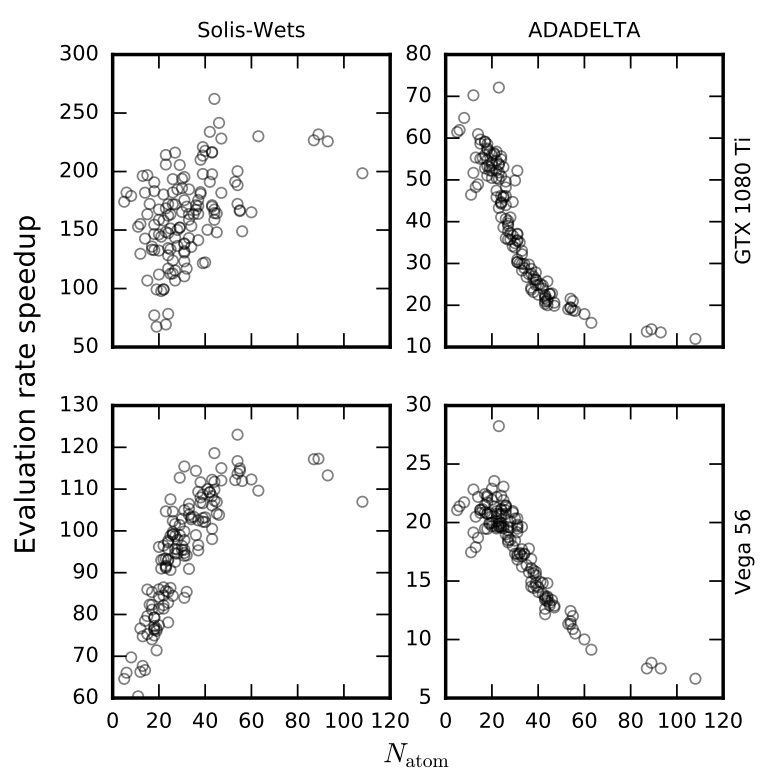

Fig. 10. Evaluation rate speedup of AUTODOck-GPU (on GTX $1080 \mathrm{Ti}$ and Vega 56 GPUs) over AUTODock4 (on a single CPU core of an AWS c5.18x instance). Note that each subplot has a different scale for the $y$-axis.

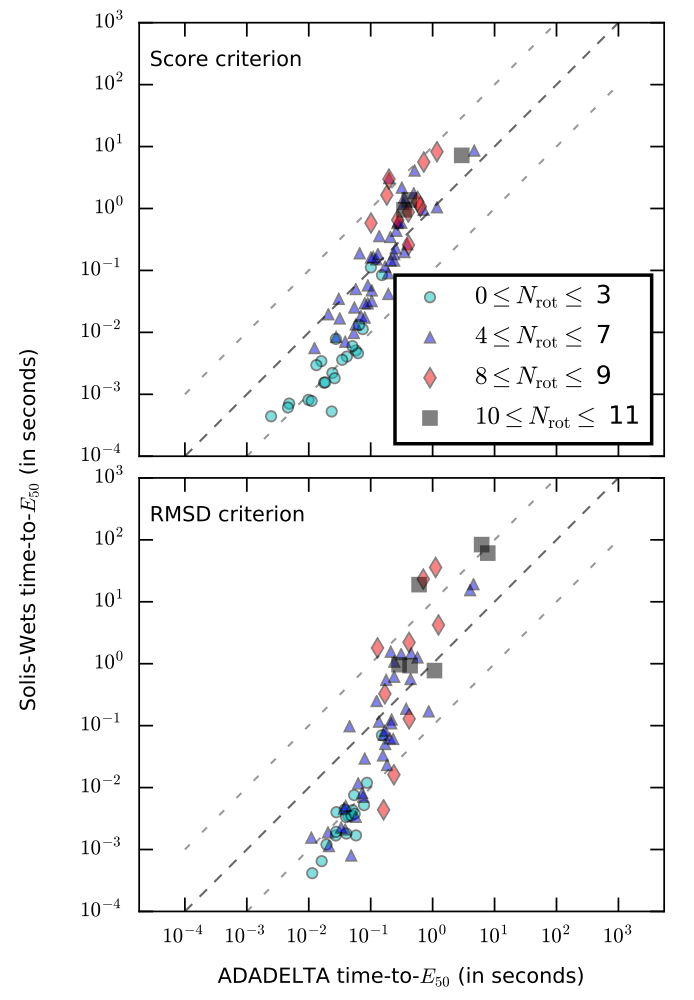

Fig. 11. Direct comparison of time-to- $E_{50}$ values between ADADELTA and SolisWets using $100 \%$ LS rate. For a given protein-ligand complex, time-to- $E_{50}$ is the product of $E_{50}$ by the corresponding evaluation rate ( $\mu \mathrm{s} /$ evaluation). Here, evaluation rates were collected on the Vega 56 GPU platform.

be developed to automatically identify convergence (e.g., AUTODockFR (39)), and hence, reduce the overall compu- tation time by preventing unnecessary evaluations from being performed past the point of convergence. In that scenario, the time-to- $E_{50}$ could be a good estimator for the time-tocompletion of docking.

Runtime performance. Here, we report the achieved runtime speedups of AUTODOCK-GPU (on various GPUs) with respect to AUTODocK4 (on a single CPU core). This analysis is based on the time-to-completion, understood as the total wall clock time spent by the docking program. The corresponding runtime speedups are therefore slightly different from evaluation rate speedups reported in a previous section. While score evaluations account for the generally most time-consuming part of docking, there are other tasks, such as loading input files or clustering LGA final poses, that are not accounted for by the evaluation rate. For this reason, reporting runtime speedups is complementary to evaluation rate speedups.

For this analysis, we selected five different ligand-receptor complexes with different number of atoms $\left(N_{\text {atom }}\right)$ and rotatable bonds $\left(N_{\text {rot }}\right)$. Although this set is small, it is yet representative for the purpose of this section as it provides enough variety of low (PDB ID: 5tim), medium (PDB ID: 2bm2, 5wlo), and high (PDB ID: 4er4, 3er5) molecular size and complexity.

The results are presented in Figure 12. Overall, Solis-Wets leads to higher runtime speedups than ADADELTA, in agreement with the previously discussed evaluation rate speedups. The better the GPU card, the higher the runtime speedups, with a nearly 10x difference observed between the M2000 and the GTX 1080 Ti GPUs. For Solis-Wets, ligands with more atoms yield higher speedups, while the opposite trend is observed for ADADELTA. This is in agreement with the severe evaluation rate slowdown observed for ADADELTA with increased number of atoms. However, while a SolisWets docking will finish faster for a given number of evaluations, ADADELTA may return significantly better poses, both in terms of score and RMSD, due to its higher algorithmic performance for ligands with many rotatable bonds, especially eight or more.

Leveraging OpenCL portability. A key feature of OpenCL is its portability. This allows running applications - ideally with none or only minor recoding effort - on different devices. In this study, this feature was leveraged by using multicore CPUs as execution platform in addition to GPUs. Our implementation ensures that the porting effort from GPUs to CPUs is reduced to just a re-compilation step, i.e., re-building the application targeting a different device architecture, but requiring no code changes.

Performance scaling on multicore CPUs. Performance scaling refers to the performance enhancement due to an increase of computing resources. This is typically translated into shorter runtimes, and hence, into lower costs for such resources. In order to assess the resource investment in terms of 
Table 4. Hardware characteristics of evaluated CPU-based platforms. Prices comprise the basic cost of compute services only (40) (i.e., underlying servers in the EU zone), and do not include charges for additional services (e.g., storage, database, migration, etc). In addition, as suggested for high-performance workloads (41), hyperthreading was disabled, allowing only a single thread per CPU core.

\begin{tabular}{|c|c|l|l|l|c|c|}
\hline $\begin{array}{c}\text { AWS } \\
\text { CPU-based } \\
\text { platform }\end{array}$ & $\begin{array}{l}\text { Number } \\
\text { of } \\
\text { cores }\end{array}$ & $\begin{array}{l}\text { Peak } \\
\text { Memory } \\
\text { Bandwidth } \\
\text { (GB/s) }\end{array}$ & $\begin{array}{l}\text { Peak Compute } \\
\text { Performance } \\
\text { FP32 } \\
\text { (GFLOPS/s) }\end{array}$ & $\begin{array}{l}\text { Total } \\
\text { RAM } \\
\text { Memory } \\
\text { (GB) }\end{array}$ & $\begin{array}{l}\text { On-demand } \\
\text { EC2 price } \\
\text { EU zone } \\
\text { (\$/hour) }\end{array}$ & $\begin{array}{l}\text { Release } \\
\text { Year }\end{array}$ \\
\hline c5.4xlarge & 8 & 42 & 768 & 30 & 0.776 & 2017 \\
\hline c5.9xlarge & 16 & 85 & 1536 & 70 & 1.746 & 2017 \\
\hline c5.18xlarge & 36 & 128 & 3456 & 140 & 3.492 & 2017 \\
\hline
\end{tabular}

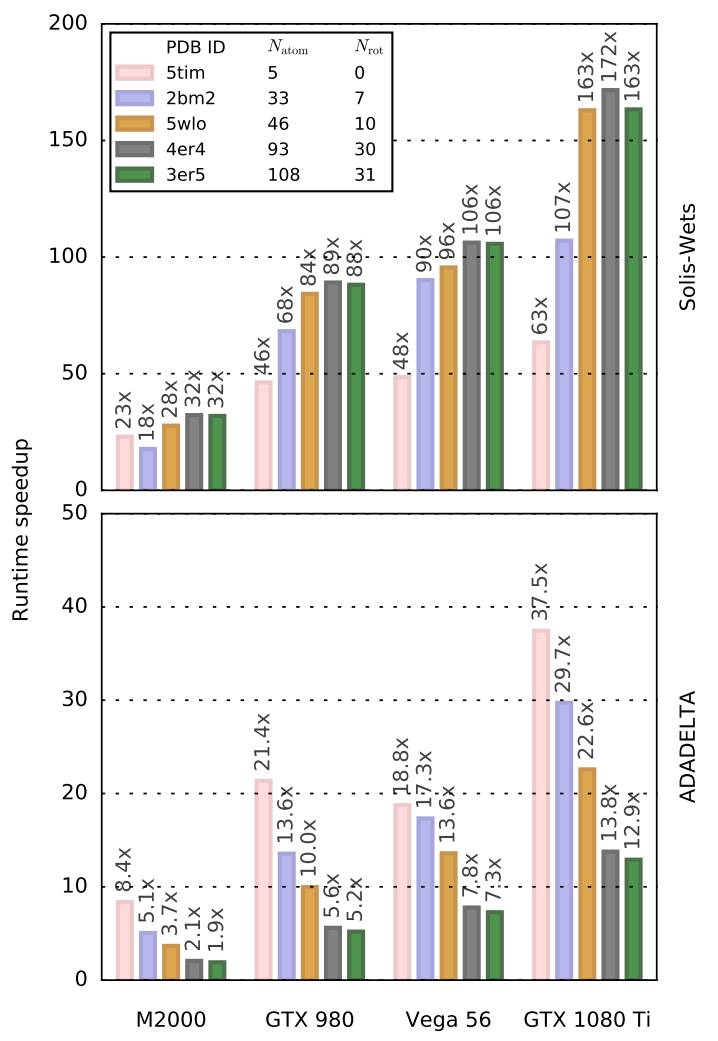

Fig. 12. Runtime speedups of AUTODOck-GPU (on various GPUs) with respect to AUTODOck4 on a single CPU core. The number of LGA runs is 100 , and the number of evaluations is $2,048,000$.

the returned performance gains, we discuss the performance scaling behavior of AUTODocK-GPU on multicore CPUs.

To do that, we selected AWS CPU instances equipped with different numbers of cores - e.g., c $5.4 \times$ large ( 8 cores), c5. $9 \times$ large (16 cores), and C $5.18 \times$ large (36 cores) - the latter being the same CPU platform used in previous experiments (only a single CPU core was used for AutoDock4). This analysis was performed on the same five complexes used for reporting GPU runtime speedups (Figure 12).

In contrast to GPUs, where the overall performance when running Solis-Wets increases along with $N_{\text {atom }}$ (Figure 12), multicore CPUs yield lower runtime performance for larger $N_{\text {atom }}$ values (Figure 13). For instance, on an 8-core instance, Solis-Wets achieves $\sim 12 \mathrm{x}$ of runtime speedup with the smallest complex (PDB ID: 5tim), while only reaching $\sim 2 \mathrm{x}$ with the largest one (PDB ID: 3er5). We attribute the different speedup dependencies on $N_{\text {atom }}$ and $N_{\text {rot }}$ values between multicore CPU and GPU to the considerably larger number of fine-grained computing cores on GPUs (e.g., 3584 CUDA cores on a GTX $1080 \mathrm{Ti}$ GPU) that more efficiently leverage the larger fine-grained parallelism - provided by more atoms and rotatable bonds - than by using fewer (here: eight) multicore CPU cores (e.g., a maximum of 36 cores on the AWS c5.18xlarge instance).

From the numbers in Figure 13, it can be noted that speedups increase with a factor of $\sim 2 \mathrm{x}$ when e.g., migrating from an instance with eight cores (c5.4xlarge) to another with 16 cores (c5.9xlarge). Slightly higher runtime speedup gains are observed when migrating to the largest c5.18xlarge instance, which can be simply explained by the upgrade provided by having more than double the number of cores $(=36)$. A similar speedup scaling behavior was observed when running ADADELTA. Additionally, Figure 13 depicts the resource-utilization efficiency, calculated as the ratio between the runtime speedup and the number of CPU cores present in the employed AWS CPU instances. For each LS method, all CPU instances achieve similar resource-utilization efficiencies, which are higher when no rotatable bonds are present ( $5 \mathrm{tim}$ ), achieving $\sim 1.4 \mathrm{x}$ and $\sim 0.75 \mathrm{x}$ when using Solis-Wets and ADADELTA, respectively. For the other complexes (having more than seven rotatable bonds), such efficiencies become lower but more stable, i.e., $\sim 0.3 \mathrm{x}$ and $\sim 0.2 \mathrm{x}$ when using Solis-Wets and ADADELTA, respectively.

While the larger AWS CPU instance (c5.18xlarge) provided the highest runtime speedup among all multicore CPUs employed, it is relevant to contextualize its performance when running AUTODocK-GPU by comparing it against high-end GPUs offered by AWS as well. For that purpose, we considered one of the latest AWS GPU-based machines - i.e., a p3.2xlarge instance - equipped with a single Volta (V100) GPU card, and costing 3.823 \$/hour in the EU zone (40). Figure 14 shows the much higher runtime speedups achieved on the p3.2xlarge instance (a maximum of $\sim 367 \mathrm{x}$ on $4 \mathrm{er} 4$ ) compared to those of c5.18xlarge (also shown in Figure 13). Furthermore, Figure 14 compares the economic efficiency yielded on both instances. This latter metric, calculated as the ratio between the runtime speedups and their prices, shows that despite the slightly higher price-per-hour of $\mathrm{p} 3.2 \times$ large (3.823 \$/hour) with respect to that of $c 5.18 \times 1$ arge (3.456 $\$$ /hour) - the returned gains in terms of the runtime 

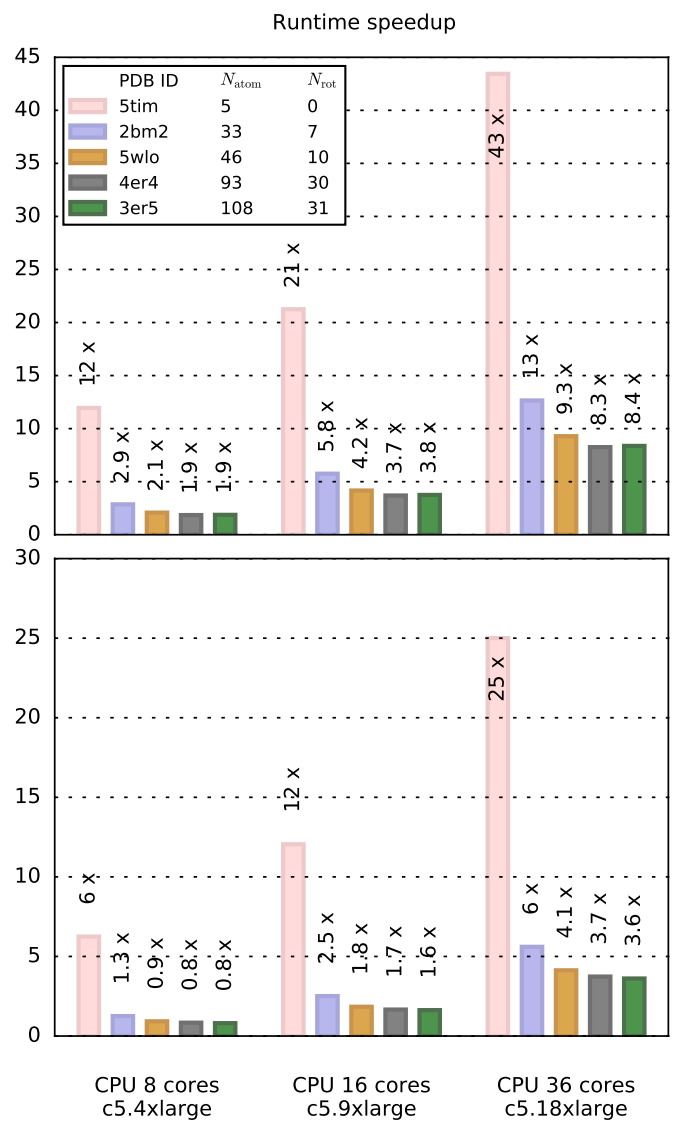
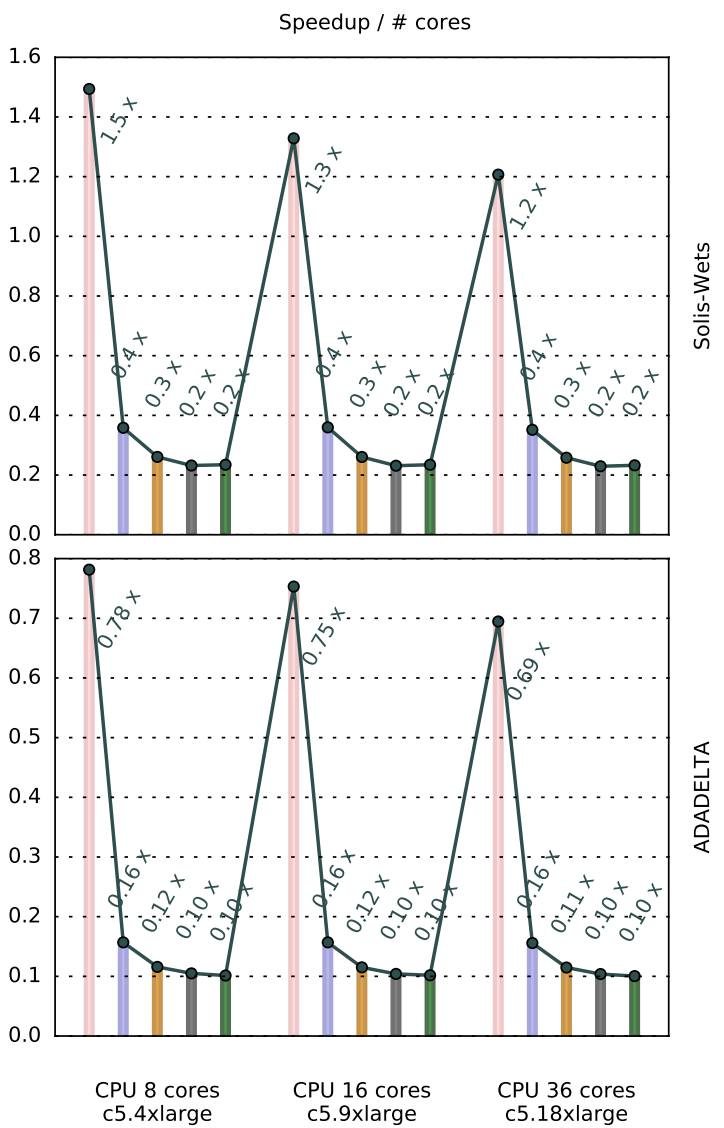

Fig. 13. Performance scalability (left) and resource-utilization efficiency (right), both in terms of the number of CPU cores. The number of LGA runs is 100 , and the number of evaluations is $2,048,000$. For each ligand-receptor complex, same program commands were executed on all three CPU instances.

speedup are always higher for GPUs than for CPUs, when using both LS methods. Therefore, it is worth investing on larger machines (having more computing resources) for executing AUTODock-GPU.

Porting AutoDock to other hardware accelerators. The functional portability of OpenCL can be exploited further. For instance, porting AUTODOCK-GPU onto emerging OpenCL-capable accelerators (which might lack driver support from the vendor) would be feasible by using opensource implementations such as POCL (42). Moreover, in recent years, OpenCL has gained increasing attention from manufacturers of highly reconfigurable accelerators such as Field Programmable Gate Arrays (FPGAs). Development tools and compilers for OpenCL on FPGAs are becoming more competitive to those of GPUs and CPUs, and are closing the gap between all these technologies. Although performance portability is still a known issue of OpenCL, our previous study of AUTODOCK-GPU on FPGAs (43) shows that this can be mitigated (up to a certain extent) with more specialized programming styles and patterns.

\section{CONCLUSIONS}

This work describes AUTODOCK-GPU, an OpenCLaccelerated version of AUTODOCK4 running on GPUs, enhanced with the ADADELTA gradient-based method used for local search. We observed improvements of both algorithmic performance as well as in the evaluation rate.

Algorithmic performance refers to the number of score evaluations required to achieve a pre-defined level of solution quality. This level is based on the score defined by the AUTODOCK 4 scoring function, together with the structural quality of docked poses (measured by the RMSD from the native pose). Based on our experiments, the algorithmic improvement of ADADELTA over Solis-Wets increases with the number of rotatable bonds. For a ligand with three or four rotatable bonds, the algorithmic performance of both local-search methods is similar. For a ligand with 12 rotatable bonds, ADADELTA finds correct solutions while performing only 1/50th of evaluations than Solis-Wets. For a ligand with 20 rotatable bonds, we estimate this reduction to increase to 1/1300. Algorithmic improvements of this magnitude can be the difference between a successful and a failed docking.

The evaluation rate is the time spent (in $\mu$ s) per score evaluation. On GPUs, the time spent in a single ADADELTA evaluation is $4 \mathrm{x}-16 \mathrm{x}$ longer than in one Solis-Wets evaluation. This is because ADADELTA requires the calculation of gradients, which is computationally more expensive.Therefore, for ligands with a small number of rotatable bonds, the algorithmic advantage of ADADELTA does not outweigh its increased computational cost. Taking into account both the time spent per evaluation, as well as the number of evalua- 

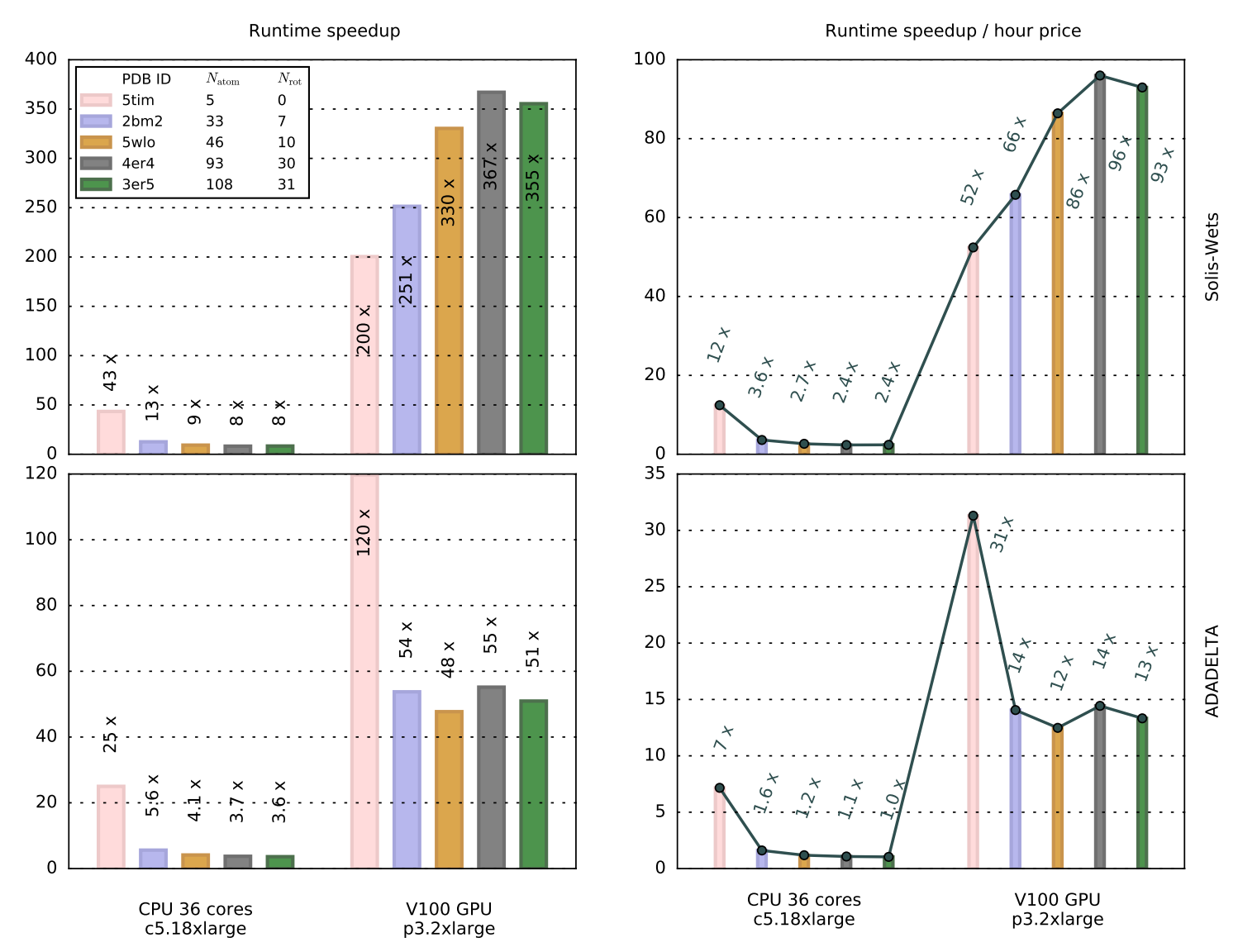

Fig. 14. Comparison of runtime speedup (left) and economic efficiency (right) between CPU and GPU instances on AWS: c $5.18 \times 1$ arge and $\mathrm{p} 3.2 \mathrm{xlarge}$, respectively. The number of LGA runs is 100 , and the number of evaluations is $2,048,000$. For each ligand-receptor complex, same program commands were executed on both instances.

tions required to produce correct solutions, we estimate that Solis-Wets is better for ligands with seven or fewer rotatable bonds, while ADADELTA is better for ligands with eight or more rotatable bonds.

Regarding runtime improvements, AuTODocK-GPU achieves speedups ranging between $\sim 2 \mathrm{x}$ and $\sim 170 \mathrm{x}$ on GPUs, depending on the hardware specifications of the accelerator platform, and the local-search method. The number of ligand atoms also affects runtime speedups, with larger ligands yielding higher speedups for Solis-Wets, but lower speedups for ADADELTA. Interestingly, if the accelerator platform is a multicore CPU, more ligand atoms always cause a lower speedup, independently of the local-search method used. This is probably due to less effective parallelization of fine-grained tasks on CPUs, with their reduced number (compared to GPUs) of available processing elements (cores). We found that such tasks can be parallelized more effectively on GPUs, with their many (in the order of thousands) fine-grained processing elements.

The accelerator platforms tested in this study were commercial GPUs from different vendors (Nvidia \& AMD) that ranged from low- to high-end devices. Using GPUs with such different computing capabilities allows potential users to estimate the performance of AUTODOCK-GPU on systems similar to those available at their sites. Furthermore, we leveraged the portability of OpenCL by using AuTODock-GPU on general-purpose multicore CPUs and showing that it is still able to provide scalable performance gains. This could be beneficial in research settings where high-end GPUs are not available.

Finally, the improvements reported here are very large in terms of improved computational efficiency. Although other docking programs exist that implement gradient-based localsearch methods (e.g., AUTODocK VINA), this work provides a much faster computation of the AUTODOCK4 scoring function, adding a new highly efficient tool to the toolbox of medicinal chemists.

ACKNOWLEDGMENTS. This work was supported by the National Institutes of Health GMO69832 (to SF), the AWS Cloud Credits for Research program, and by the ALEPRONA funding program \#57186883 from the German Academic Exchange Service (DAAD) and the Peruvian National Program for Scholarships and Educational Loans (PRONABEC).

We thank JC Ducom and the HPC facility of The Scripps Research Institute, as well as AMD Inc. for technical support. We acknowledge the use of GNU Parallel (44), Matplotlib (45) and Seaborn (46).

AVAILABILITY. AUTODock-GPU is open source under a GPL licence. Its source code as well as its documentation 
is available at:

https: / / autodock.scripps.edu

https://github.com/ccsb-scripps/AutoDock-GPU

\section{REFERENCES}

1. Brian K. Shoichet. Virtual screening of chemical libraries. Nature, 432:862-865, 2004.

2. John J. Irwin and Brian K. Shoichet. Zinc - a free database of commercially available compounds for virtual screening. J. Chemical Information and Modeling, 45(1):177-182, 2005.

3. Brian K. Shoichet. Screening in a spirit haunted world. J. Drug Discovery Today, 11(13): 607-615, 2006.

4. Brian K. Shoichet and Brian K. Kobilka. Structure-based drug screening for g-proteincoupled receptors. J. Trends in Pharmacological Sciences, 33(5):268 - 272, 2012.

5. Virginia A. Kincaid, Nir London, Kittikhun Wangkanont, Darryl A. Wesener, Sarah A. Marcus, Annie Héroux, Lyudmila Nedyalkova, Adel M. Talaat, Katrina T. Forest, Brian K. Shoichet, and Laura L. Kiessling. Virtual screening for udp-galactopyranose mutase ligands identifies a new class of antimycobacterial agents. J. ACS Chemical Biology, 10(10):2209-2218, 2015.

6. Marc-André Elsliger, Ashley M. Deacon, Adam Godzik, Scott A. Lesley, John Wooley, Kurt Wüthrich, and lan A. Wilson. The jcsg high-throughput structural biology pipeline. J. Acta Crystallographica Section F, 66(10):1137-1142, 2010.

7. Jung-Hsin Lin, Alexander L. Perryman, Julie R. Schames, and J. Andrew McCammon. The relaxed complex method: Accommodating receptor flexibility for drug design with an improved scoring scheme. J. Biopolymers, 68(1):47-62, 2003.

8. Oleg Trott and Arthur J. Olson. Autodock vina: Improving the speed and accuracy of docking with a new scoring function, efficient optimization, and multithreading. J. Computational Chemistry, 31(2):455-461, 2010.

9. Stefano Forli, Ruth Huey, Michael E. Pique, Michel F. Sanner, David S. Goodsell, and Arthur J. Olson. Computational protein-ligand docking and virtual drug screening with the autodock suite. Nature Protocols, 11:905-919, 2016.

10. David P. Anderson. Boinc: A system for public-resource computing and storage. In Proceedings of the 5th IEEE/ACM International Workshop on Grid Computing, Washington, DC, USA, 2004. IEEE Computer Society.

11. World Community Grid. https://www. worldcommunitygrid.org. accessed January $30,2019$.

12. Daniel J. Mermelstein, Charles Lin, Gard Nelson, Rachael Kretsch, J. Andrew McCammon, and Ross C. Walker. Fast and flexible gpu accelerated binding free energy calculations within the amber molecular dynamics package. J. Computational Chemistry, 39(19):1354$1358,2018$.

13. John E. Stone, David J. Hardy, Jan Saam, Kirby L. Vandivort, and Klaus Schulten. Chapter 1 - gpu-accelerated computation and interactive display of molecular orbitals. In GPU Computing Gems Emerald Edition, Applications of GPU Computing Series, pages 5-18. Morgan Kaufmann, 2011.

14. John E. Stone, Antti-Pekka Hynninen, James C. Phillips, and Klaus Schulten. Early experiences porting the namd and vmd molecular simulation and analysis software to gpuaccelerated openpower platforms. In High Performance Computing, Cham, 2016. Springer

15. Sparsh Mittal and Jeffrey S. Vetter. A survey of cpu-gpu heterogeneous computing techniques. ACM Computing Surveys, 47(4):69:1-69:35, 2015

16. Evgenij Belikov, Pantazis Deligiannis, Prabhat Totoo, Malak Aljabri, and Hans-Wolfgang Loidl. A survey of high-level parallel programming models. Technical Report HW-MACS TR-0103, 2013.

17. J. Diaz, C. Muñoz-Caro, and A. Niño. A survey of parallel programming models and tools in the multi and many-core era. IEEE Transactions on Parallel and Distributed Systems, 23 (8): 1369-1386, 2012

18. Garrett M. Morris, Ruth Huey, William Lindstrom, Michel F. Sanner, Richard K. Belew, David S. Goodsell, and Arthur J. Olson. Autodock4 and autodocktools4: Automated docking with selective receptor flexibility. J. Computational Chemistry, 30(16):2785-2791, 2009.

19. Matthew D. Zeiler. ADADELTA: an adaptive learning rate method. arXiv, abs/1212.5701, 2012.

20. Francisco J. Solis and Roger J. B. Wets. Minimization by random search techniques. J. Mathematics of Operations Research, 6(1):19-30, 1981.

21. Ruth Huey, Garrett M. Morris, Arthur J. Olson, and David S. Goodsell. A semiempirica free energy force field with charge-based desolvation. J. Computational Chemistry, 28(6): 1145-1152, 2007.

22. Garrett M. Morris, David S. Goodsell, Robert S. Halliday, Ruth Huey, William E. Hart Richard K. Belew, and Arthur J. Olson. Automated docking using a lamarckian genetic algorithm and an empirical binding free energy function. J. Computational Chemistry, 19 (14):1639-1662, 1998.

23. Leonardo Solis-Vasquez and Andreas Koch. A performance and energy evaluation of opencl-accelerated molecular docking. In Proceedings of the 5th International Workshop on OpenCL, New York, NY, USA, 2017. ACM.

24. OCLADock - OpenCL Accelerated Molecular Docking. https://git.esa. informatik.tu-darmstadt.de/docking/ocladock. accessed October 10 2018.

25. The OpenCL Specification. https://www. khronos.org/opencl. accessed October 10,2018

26. Imre Pechan and Bela Feher. Molecular docking on fpga and gpu platforms. In Proceedings of the 21st International Conference on Field Programmable Logic and Applications. IEEE, 2011.

27. Imre Pechan, Béla Fehér, and Attila Bérces. Fpga-based acceleration of the autodock molecular docking software. In Proceedings of the 6th Conference on Ph.D. Research in Microelectronics Electronics. IEEE, 2010.
28. OpenCL 2.0 Reference Pages. https://www.khronos.org/registry/OpenCL/ $\mathrm{sdk} / 2.0 / \mathrm{docs} / \mathrm{man} / \mathrm{xhtml}$, accessed October 10, 2018.

29. Jinghui Zhong, Xiaomin Hu, Jun Zhang, and Min Gu. Comparison of performance between different selection strategies on simple genetic algorithms. In Proceedings of the International Conference on Computational Intelligence for Modelling, Control and Automation and International Conference on Intelligent Agents, Web Technologies and Internet Commerce. IEEE, 2005.

30. Noraini Mohd Razali and John Geraghty. Genetic algorithm performance with different selection strategies in solving tsp. In World Congress on Engineering. IAENG, 2011.

31. Jorge Nocedal and Stephen J. Wright. Numerical Optimization. Springer, 2 edition, 2006

32. Diederik P Kingma and Jimmy Ba. Adam: A method for stochastic optimization. arXiv, abs/1412.6980, 2014

33. Erik Bitzek, Pekka Koskinen, Franz Gähler, Michael Moseler, and Peter Gumbsch. Structural relaxation made simple. J. Physical Review Letters, 97(17):170201, 2006.

34. Michael J. Hartshorn, Marcel L Verdonk, Gianni Chessari, Suzanne C Brewerton, Wijnand TM Mooij, Paul N Mortenson, and Christopher W Murray. Diverse, high-quality test set for the validation of protein- ligand docking performance. J. Medicinal Chemistry, 50(4): 726-741, 2007.

35. Yan Li, Li Han, Zhihai Liu, and Renxiao Wang. Comparative assessment of scoring functions on an updated benchmark: 2. evaluation methods and general results. J. Chemical Information and Modeling, 54(6):1717-1736, 2014.

36. Helen M. Berman, John Westbrook, Zukang Feng, Gary Gilliland, T. N. Bhat, Helge Weissig, Ilya N. Shindyalov, and Philip E. Bourne. The protein data bank. J. Nucleic Acids Research, 28(1):235-242, 2000

37. Noel M. O'Boyle, Michael Banck, Craig A. James, Chris Morley, Tim Vandermeersch, and Geoffrey R. Hutchison. Open babel: An open chemical toolbox. J. Cheminformatics, 3(1): 33, 2011

38. Nikolaus Hansen, Anne Auger, Raymond Ros, Steffen Finck, and Petr Pošík. Comparing results of 31 algorithms from the black-box optimization benchmarking bbob-2009. In Proceedings of the 12th Annual Conference Companion on Genetic and Evolutionary Com putation, New York, NY, USA, 2010. ACM.

39. Pradeep A. Ravindranath, Stefano Forli, David S. Goodsell, Arthur J. Olson, and Michel F. Sanner. Autodockfr: Advances in protein-ligand docking with explicitly specified binding site flexibility. J. PLOS Computational Biology, 11(12):1-28, 2015.

40. Amazon EC2 Pricing. https://aws.amazon.com/ec2/pricing/on-demand/, accessed January 30, 2019

41. Amazon Elastic Compute Cloud, User Guide for Linux Instances. https ://docs . aws amazon.com/AWSEC2/latest/UserGuide/instance-optimize-cpu.html, accessed January 30, 2019.

42. Pekka Jääskeläinen, Carlos Sánchez de La Lama, Erik Schnetter, Kalle Raiskila, Jarmo Takala, and Heikki Berg. pocl: A performance-portable opencl implementation. Int. J. of Parallel Programming, 43(5):752-785, 2015.

43. Leonardo Solis-Vasquez and Andreas Koch. A case study in using opencl on fpgas: Creating an open-source accelerator of the autodock molecular docking software. In Proceedings of the 5th International Workshop on FPGAs for Software Programmers, Berlin, Germany, 2018. VDE Verlag GmbH.

44. O. Tange. Gnu parallel - the command-line power tool. ;login: The USENIX Magazine, 36 (1):42-47, 2011.

45. J. D. Hunter. Matplotlib: A 2d graphics environment. Computing in Science \& Engineering, 9(3):90-95, 2007. doi: 10.1109/MCSE.2007.55.

46. Seaborn, $10.5281 /$ zenodo.592845.

\section{APPENDIX}

Table 5. Upper limits on AUTODOCK-GPU docking parameters (defined in /common/defines.h).

\begin{tabular}{|c|c|c|}
\hline $\begin{array}{l}\text { Upper limit } \\
\text { identifier }\end{array}$ & $\begin{array}{c}\text { Description (as } \\
\text { maximum number of) }\end{array}$ & Value \\
\hline ATYPE_NUM & $\begin{array}{l}\text { Ligand atomic types } \\
\text { for smoothing }\end{array}$ & 22 \\
\hline MAX_NUM_OF_ATOMS & Ligand atoms & 256 \\
\hline MAX_NUM_OF_ATYPES & $\begin{array}{l}\text { Ligand atomic types } \\
\text { for scoring function }\end{array}$ & 14 \\
\hline MAX_NUM_OF_ROTBONDS & Rotatable bonds & 32 \\
\hline MAX_INTRAE_CONTRIBUTORS & $\begin{array}{l}\text { Intramolecular (pairwise) } \\
\text { energy contributors }\end{array}$ & $\begin{array}{c}\text { MAX_NUM_OF_ATOMS * } \\
\text { MAX_NUM_OF_ATOMS }\end{array}$ \\
\hline MAX_NUM_OF_ROTATIONS & $\begin{array}{l}\text { Rotations } \\
\text { to be performed }\end{array}$ & $\begin{array}{l}\text { MAX_NUM_OF_ATOMS * } \\
\text { MAX_NUM_OF_ROTBONDS }\end{array}$ \\
\hline MAX_POPSIZE & Individuals in a population & 2048 \\
\hline MAX_NUM_OF_RUNS & Docking runs & 1000 \\
\hline MAX_NUM_GRIDPOINTS & Grid points per dimension & 256 \\
\hline
\end{tabular}


Table 6. Constant data structures and their members in AUTODOCK-GPU.

\begin{tabular}{|c|c|c|c|c|c|}
\hline $\begin{array}{c}\text { Struct } \\
\text { label }\end{array}$ & $\begin{array}{l}\text { Constant array } \\
\text { struct member }\end{array}$ & $\begin{array}{l}\text { Element } \\
\text { datatype }\end{array}$ & $\begin{array}{c}\text { Size } \\
\text { definition }\end{array}$ & $\begin{array}{c}\text { Size } \\
\text { calculation }\end{array}$ & $\begin{array}{c}\text { Size } \\
\text { (Bytes) }\end{array}$ \\
\hline$\overline{\mathrm{A}}$ & $\begin{array}{l}\text { atom_charges } \\
\text { atom_types }\end{array}$ & $\begin{array}{l}\text { float } \\
\text { char }\end{array}$ & $\begin{array}{l}\text { MAX_NUM_OF_ATOMS } \\
\text { MAX_NUM_OF_ATOMS }\end{array}$ & $\begin{array}{l}4 * 256 \\
1 * 256\end{array}$ & $\begin{array}{l}1024 \\
256\end{array}$ \\
\hline$\overline{\mathrm{B}}$ & intraE_contributors & char & 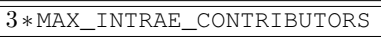 & $1 * 3 * 256 * 256$ & 196608 \\
\hline$\overline{\mathrm{C}}$ & $\begin{array}{c}\text { reqm } \\
\text { reqm_hbond } \\
\text { atom1_types_reqm } \\
\text { atom2_types_reqm } \\
\text { VWpars_AC } \\
\text { VWpars_BD } \\
\text { dspars_s } \\
\text { dspars_V }\end{array}$ & $\begin{array}{l}\text { float } \\
\text { float } \\
\text { unsigned int } \\
\text { unsigned int } \\
\text { float } \\
\text { float } \\
\text { float } \\
\text { float }\end{array}$ & $\begin{array}{c}\text { ATYPE_NUM } \\
\text { ATYPE_NUM } \\
\text { ATYPE_NUM } \\
\text { ATYPE_NUM } \\
\text { (MAX_NUM_OF_ATYPES * } \\
\text { MAX_NUM_OF_ATYPES) } \\
\text { (MAX_NUM_OF_ATYPES * } \\
\text { MAX_NUM_OF_ATYPES) } \\
\text { MAX_NUM_OF_ATYPES } \\
\text { MAX_NUM_OF_ATYPES }\end{array}$ & $\begin{array}{c}4 * 22 \\
4 * 22 \\
4 * 22 \\
4 * 22 \\
4 * 14 * 14 \\
4 * 14 * 14 \\
4 * 14 \\
4 * 14\end{array}$ & $\begin{array}{c}88 \\
88 \\
88 \\
88 \\
784 \\
\\
784 \\
\\
\\
56 \\
56\end{array}$ \\
\hline $\mathrm{D}$ & rotlist & int & PAX_NUM_OF_ROTATIONS & $4 * 256 * 32$ & 32768 \\
\hline $\bar{E}$ & $\begin{array}{c}\text { ref_coords_x } \\
\text { ref_coords_y } \\
\text { ref_coords_z } \\
\text { rotbonds_moving_vectors } \\
\text { rotbonds_unit_vectors } \\
\text { ref_orientation_quats }\end{array}$ & $\begin{array}{l}\text { float } \\
\text { float } \\
\text { float } \\
\text { float } \\
\text { float } \\
\text { float }\end{array}$ & $\begin{array}{c}\text { MAX_NUM_OF_ATOMS } \\
\text { MAX_NUM_OF_ATOMS } \\
\text { MAX_NUM_OF_ATOMS } \\
3 * \text { MAX_NUM_OF_ROTBONDS } \\
3 * \text { MAX_NUM_OF_ROTBONDS } \\
4 * \text { MAX_NUM_OF_RUNS }\end{array}$ & $\begin{array}{c}4 * 256 \\
4 * 256 \\
4 * 256 \\
4 * 3 * 32 \\
4 * 3 * 32 \\
4 * 4 * 1000 \\
\end{array}$ & $\begin{array}{c}1024 \\
1024 \\
1024 \\
384 \\
384 \\
16000 \\
\end{array}$ \\
\hline & & & & Subtotal size (Bytes) & 252528 \\
\hline GRIDS & fgrids & float & $\begin{array}{l}\text { MAX_NUM_OF_ATYPES * } \\
\text { MAX_NUM_GRIDPOINTS }{ }^{3}\end{array}$ & $4 * 16 * 256^{3}$ & 1073741824 \\
\hline & & & & Total size (Bytes) & 1073994352 \\
\hline
\end{tabular}

Table 7. Additional constant data structures and their members for gradient calculation in AUTODOCK-GPU.

\begin{tabular}{|c|c|c|c|c|c|}
\hline $\begin{array}{c}\text { Struct } \\
\text { label }\end{array}$ & $\begin{array}{c}\text { Constant array } \\
\text { struct member }\end{array}$ & $\begin{array}{c}\text { Element } \\
\text { datatype }\end{array}$ & $\begin{array}{c}\text { Size } \\
\text { definition }\end{array}$ & $\begin{array}{c}\text { Size } \\
\text { calculation }\end{array}$ & $\begin{array}{c}\text { Size } \\
\text { (Bytes) }\end{array}$ \\
\hline \hline $\mathrm{F}$ & rotbonds_atoms & int & MAX_NUM_OF_ATOMS* & $4 * 256 * 32$ & 32768 \\
& & & MAX_NUM_OF_ROTBONDS & \\
\hline \hline $\mathrm{G}$ & rotbonds & int & $2 *$ MAX_NUM_OF_ROTBONDS & $4 * 2 * 32$ & 256 \\
& num_rotating_atoms_per_rotbond & int & MAX_NUM_OF_ROTBONDS & $4 * 32$ & 128 \\
& angle & float & 1000 & $4 * 1000$ & 4000 \\
& float & 1000 & $4 * 1000$ & 4000 \\
& dependence_on_rotangle & float & 1000 & $4 * 1000$ & 4000 \\
\hline \hline & & & & Total size (Bytes) & 45152 \\
\hline
\end{tabular}

